\title{
Cosmological Concepts Astrophysical Extension of Non-Interactive Mechanics
}

\author{
Nishant Kumar Sharma \\ Gadarpur, Uttrakhand, India \\ Author's Mailid:nkphysics11@gmail.com
}

Available online at: www.isroset.org

Received: 24/Dec/2019, Accepted: 22/Jan/2020, Online: 28/Feb/2020

\begin{abstract}
In This Manuscript We Will Study About The Condition For Creation Of Non-Reacting Zero Space And Creation Of Always Reacting Infinite Space. Then We Will Study About The Infinite River Of Quant's, Then The Creation Of Space And Time By This River Of Quantum Domains, The Creation Of Space And Time In Universe Is Depended on The Adjustment Of Quantum Domains In Previously Created Space-Time, condition for space creation and it's formula With Satisfying The Condition, condition for appearance of time With Satisfying The Condition Theoretically And Experimentally, Quantum Mechanical System Aatmamoul for the movement of Elementary And Non-Intractable particles in Physical And Universal Bodies, concept of Adjustment Of Quantum Domains. concepts of app. Stable systems (planets ,Stars) ,concept of consciousness, Then There is Discussion About Gravitation The Emergent Phenomena From Elementary and Non-Intractable Particles, How Newton To Einstein We Are On The Correct Path Of Gravitation And How Einstein Was Right About Cosmological Constant Even When Universe Is Expanding And Why We Need An Extension To Understand Gravitation Correctly, What Is Gravitation And How It Originate , Types Of Gravitation And Gravitational Waves, The Test About Contracting Gravitation That Could Be or May Be already detected In Ligo, The Equations To Describe The Behavior Of Gravitation, The Combined Field Equation Of Gravitation For Expanding And Contraction Nature With Satisfying The Equation, Conditions To Find Graviton.
\end{abstract}

Keywords- Condition for time appearance and space creation, Para-Kans, Field Equation of Gravitation.

\section{INTRODUCTION}

In The Paper 'THE NON-INTERACTIVE MECHANICS' we have clearly established the relations like
$\begin{array}{ll}\text { 1. } \mathrm{N}+\mathrm{M}=0 & \text { 2. } \mathrm{N}=-\mathrm{M}\end{array}$
3. $\mathrm{N}=-\mathrm{M} \pm \mathrm{F}$ p.of
4.. $\mathrm{E}=(\mathrm{N}+\mathrm{M}) \mathrm{C}^{\wedge} 2$

And these relations satisfies the varies conditions like $1 . \mathrm{E}=1 / 2 \mathrm{hv} 2 \mathrm{E}=\mathrm{hv} 3 . E^{2}=P^{2} C^{2}+M^{2} C^{4}$

And The Second Equations N = -M Is Same As The G T R Equation G ik = -k T ik

The velocity of Non-Intractable Particles is $299813275.22 \mathrm{~m} / \mathrm{Sec}$ And the velocity of light Is $299792458 \mathrm{~m} / \mathrm{sec}$ So NonIntractable Particle Is $20817.22 \mathrm{~m} / \mathrm{sec}$ Faster Than Normal Light , this also treated as the approximation of velocity of light as we can assume that some force of friction is present in the experiment performed for gaining the velocity of light but if the system is free from the force of friction then yes! the Non-Intractable Particle is faster than the light.

According To The Special theory Of Relativity

1. All the laws of physics have the same form in all inertial systems, moving With Constant velocity relative to one another.

2. The speed of light is constant in vacuum in every inertial system.

According To Above Cases We Have To Believe That S.T.R Is Not A Complete Theory, So-

If Two Inertial Systems With The Energy Of $\mathrm{E} 1=\mathrm{M} 1 C^{2}$ and $\mathrm{E} 2=\mathrm{M} 2 C^{2}$ moving with respect to one another, when NonIntractable Particle is Not present in the Matter field Then YES The postulates of Spec. theory of relativity are correct ,but if the Non-Intractable Particle Is Present In the Matter Field Then The Above Eq. Turns As E1 $=(\mathrm{N} 1+\mathrm{M} 1) C^{2}$ and E2= $(\mathrm{N} 2+\mathrm{M} 2) C^{2}$ And these relation Satisfies The Relativistic Condition Of $E 1^{2}=P 1^{2} C^{2}+M 1^{2} C^{4}$ and $E 2^{2}=P 2^{2} C^{2}+M 2^{2} C^{4}$ with the Relation

$\mathrm{E} 1= \pm \sqrt{\mathrm{N} 1^{2}}+2 N 1 M 1+\mathrm{M} 1^{2} \cdot(c)^{2}$ And $\mathrm{E} 2= \pm \sqrt{\mathrm{N} 2^{2}+} 2 N 2 M 2+\mathrm{M} 2^{2} \cdot(c)^{2}$ and the velocity of Non-Intractable Particle is faster than the velocity of light if it is present in the Matter Field this explains few things which are-

So The Postulates Are Not Correct in All Cases. In That Situation 
1. The Laws Of Physics (Where Mass(M) is Mentioned) Have not the same form in the All Inertial systems if the Non-intractable Particle Is Present In The Matter Field.

2. The Speed Of Light Is Not A Constant In Vacuum In Any Inertial System If The Non-Intractable Particle Is Present With The Matter.

As the Not-Intractable Particle Have the Infinite Expansion Properties According To The Equation

$$
\begin{aligned}
N & =-M+0 \\
N & =-M+N+M^{\prime} \\
N & =\left(-M+M^{\prime}\right)+N \\
& =\left(-M+M^{\prime}\right)-M^{\prime \prime} \pm(f \text { p.o.f }) \quad \text { as }\left(N=-M^{\prime \prime} \pm \text { (f p.o.f }\right) \\
& =\left(-M+M^{\prime}+\mathbf{0}-M^{\prime \prime}\right) \pm(f \text { p.o.f }) \text { underlined quantity is primary source of consciousness in universe } \\
& =\left(-M+M^{\prime}+0-M^{\prime \prime}\right)+0 \\
& =\left(-M+M^{\prime}+0-M^{\prime \prime}\right)+N+M^{\prime \prime}, \text { so As More }
\end{aligned}
$$

Above Equation Defines That The Laws Of Physics Doesn't Hold The Same Form And The Velocity Of Light Is Slower Than The Velocity Of Non-Intractable Particle.

In Non-Interactive Mechanics We Deled With The Properties Of Non-Intractable Particle Such As It's Entering In Matter Field And It's Reaction With Matter Field. In This Manuscript we Will Deal With The Astronomical Properties Of NonIntractable Particles Such As The Infinite Sea Of Non-Intractable Particles, Their Role In The Formation of Space-Time, Superiority Of Space Over Time, Creation Of First Ever Triangle System, The Systems And The Mediums For The Movement Of Non-Intractable Particles ,Special Type Non-Intractable Particles The Para-Kans, The Approximately Stable Systems Created By The Varies Mediums such As Planets Stars, Consciousness And Its Particle Nature, Gravitation The Emergent Phenomena From Non-Intractable Particles, How Newton To Einstein We Are On The Correct Path Of Gravitation And Why We Need An Extension To Understand Gravitation Correctly, What Is Gravitation And How It Originate, Types Of Gravitation And Gravitational Waves, The Test About Contracting Gravitation That Could Be detected In Ligo, The Equations To Describe The Behaviour Of Gravitation, The Combined Field Equation Of Gravitation For Expanding And Contraction Nature With Satisfying The Equation, Conditions To Find Graviton.

\section{FOR CREATING A ZERO SPACE AND A INFINITY SPACE}

1. For Creating 'A Infinite View The Required Condition Is The Expansion Of Disintegrated Matter With Respect To The Study State Non-Intractable Particle' .This Gives The Infinite Expended Matter Universe.

2. For Creating 'A Zero View The Required Condition Is The Expansion Of The Non-Intractable Particles With Respected To The Constant Disintegrated Matter. This Gives The Infinite River Of Non-Intractable Particle In The Universe To Make The Union With The Matter.

Now We Will Prove Above Conditions.

1.By Non-interactive mechanics we known the equation - $\mathbf{1} . \mathbf{N}+\mathbf{M}=\mathbf{0}$ by the equation 'we know that sum of nonintractable particle and matter field is equals to class zero particle' .

Which gives the second equation that 2. $\mathbf{N}=-\mathbf{M}$, this shows 'non-intractable particle is equal to disintegrated matter' .

Now in equation $-(2)$

$\mathrm{N}=-\mathrm{M}$

$\mathrm{N}=1+2+3+\ldots \infty$

Which gives $\mathrm{N}=\frac{-1}{12}$

If $\mathrm{N}$ is the function of $\infty$

So this gives -

in equation $-(2)$

$$
\begin{gathered}
\mathbf{N}(\infty)=-\mathbf{M} \quad-2(\mathrm{a}) \\
\mathbf{N}(\infty)=\frac{-1}{12}-2(\mathrm{~b})
\end{gathered}
$$

First in equation -2 (a) $\mathbf{N}(\infty)=-\mathbf{M} \quad$ (here $\mathbf{M}=\frac{4 \pi V}{3 h^{3}} \cdot \frac{1}{4} \sqrt{\frac{8 m^{3} \pi}{\beta^{3}}} \cdot e^{\frac{\beta p^{2}}{2 m}}$ )

$$
\mathbf{N}(\infty)=\frac{4 \pi V}{3 h^{3}} \cdot \frac{1}{4} \sqrt{\frac{8 m^{3} \pi}{\beta^{3}}} \cdot e^{\frac{\beta p^{2}}{2 m}}
$$

Now multiplying $N^{-1}$ on the left of both sides we get

$N^{-1} \mathrm{~N}(\infty)=N^{-1}\left(-\frac{4 \pi V}{3 h^{3}} \cdot \frac{1}{4} \sqrt{\frac{8 m^{3} \pi}{\beta^{3}}} \cdot e^{\frac{\beta p^{2}}{2 m}}\right)$

This gives- 


$$
\infty=\frac{1}{N}\left(-\frac{4 \pi V}{3 h^{3}} \cdot \frac{1}{4} \sqrt{\frac{8 m^{3} \pi}{\beta^{3}}} \cdot e^{\frac{\beta p^{2}}{2 m}}\right)
$$

This gives that for a system $\infty=\frac{1}{N}\left(-\frac{4 \pi V}{3 h^{3}} \cdot \frac{1}{4} \sqrt{\frac{8 m^{3} \pi}{\beta^{3}}} \cdot e^{\frac{\beta p^{2}}{2 m}}\right)$ or

$$
\begin{aligned}
& \left(\infty=\frac{-M}{N}\right) \\
& \left(\infty=\frac{-\frac{4 \pi V}{3 h^{3}} \cdot \frac{1}{4} \sqrt{\frac{8 m^{3} \pi}{\beta^{3}}} \cdot e^{\frac{\beta p^{2}}{2 m}}}{N}\right)
\end{aligned}
$$

For “A Infinitely Expanded Universe ( Infinity View ) The Required Condition Is The Expansion Of Disintegrated Matter With Respect To The Study State Non-Intractable Particle “

2. Now the 0 space Or Zero View -

the equation-(2) is 2. $\mathbf{N}=-\mathbf{M}$

Taking inverse on both sides of equation $-(2) \quad(N)^{-1}=(-M)^{-1}$

As mentioned above if $\mathrm{N}$ is the function of $\infty$ than eq. -(3) can be written as

$N(\infty)^{-1}=(-M)^{-1}$

So $\frac{1}{N(\infty)}=\frac{1}{(-M)} \quad\left(\right.$ here $\mathrm{M}=\frac{4 \pi V}{3 h^{3}} \cdot \frac{1}{4} \sqrt{\frac{8 m^{3} \pi}{\beta^{3}}} \cdot e^{\frac{\beta p^{2}}{2 m}}$ )

here $\mathrm{N}$ is Non-intractable particle , and $\infty$ represents the infinite number of Non-Intractable Particles Following positive curve geometry.

$$
\frac{1}{N(\infty)}=\left(-\frac{3 h^{3}}{4 \pi V} \cdot 4 \sqrt{\frac{\beta^{3}}{8 m^{3} \pi}} \cdot \frac{1}{e^{\frac{\beta p^{2}}{2 m}}}\right)
$$

Multiplying by $\mathrm{N}$ on both sides of given equation

$$
\frac{N .1}{N(\infty)}=\mathrm{N} \cdot\left(-\frac{3 h^{3}}{4 \pi V} \cdot 4 \sqrt{\frac{\beta^{3}}{8 m^{3} \pi}} \cdot \frac{1}{e^{\frac{\beta p^{2}}{2 m}}}\right)
$$

Now multiplying $N^{-1}$ on numerator and denominator both this gives-

$$
\frac{1}{(\infty)}=\frac{N \cdot N^{-1}}{N^{-1}} \cdot\left(-\frac{3 h^{3}}{4 \pi V} \cdot 4 \sqrt{\frac{\beta^{3}}{8 m^{3} \pi}} \cdot \frac{1}{e^{\frac{\beta p^{2}}{2 m}}}\right)
$$

Which gives-

$$
\begin{aligned}
& \frac{1}{(\infty)}=\mathrm{N}\left(-\frac{3 h^{3}}{4 \pi V} \cdot 4 \sqrt{\frac{\beta^{3}}{8 m^{3} \pi}} \cdot \frac{1}{e^{\frac{\beta p^{2}}{2 m}}}\right) \\
& \text { As } \frac{1}{(\infty)}=0 \\
& \text { So - } 0=\mathrm{N}\left(-\frac{3 h^{3}}{4 \pi V} \cdot 4 \sqrt{\frac{\beta^{3}}{8 m^{3} \pi}} \cdot \frac{1}{e^{\frac{\beta p^{2}}{2 m}}}\right)
\end{aligned}
$$

So for creating the 0 space or class zero particle the required condition is

$$
\begin{aligned}
& 0=\mathrm{N}\left(-\frac{3 h^{3}}{4 \pi V} \cdot 4 \sqrt{\frac{\beta^{3}}{8 m^{3} \pi}} \cdot \frac{1}{e^{\frac{\beta p^{2}}{2 m}}}\right) \\
& 0=\mathrm{N} \cdot\left(-M^{-1}\right)
\end{aligned}
$$

$$
0=\frac{\frac{N}{(-M)}}{\left(-\frac{4 \pi V}{3 h^{3}} \cdot \frac{1}{4} \sqrt{\frac{8 m^{3} \pi}{\beta^{3}}} \cdot e^{\frac{\beta p^{2}}{2 m}}\right)}
$$

So for 'A Zero View The Required Condition Is The Expansion Of The Non-Intractable Particles With Respected To The Constant Disintegrated Matter' .

3. The concepts Of River of Quantum Domain And Required Condition Of Space Creation And The Required Condition Of Creation Of Time - Mathematical, Theoretical And Experimental Conditions

The Condition Of Space Creation-

Now by equation -2 (b)

$$
\mathbf{N}(\infty)=\frac{-1}{12}
$$

Multiplying $N^{-1}$ on both sides

$$
\begin{aligned}
N^{-1} \mathbf{N}(\infty) & =N^{-1} \cdot \frac{-1}{12} \\
(\infty) & =\frac{-1}{12 . N}-3(\text { a) } \\
\text { And if }(\infty) & =\frac{1}{0}
\end{aligned}
$$


$\frac{1}{0}=\frac{-1}{12 . N}$

Then $\frac{1}{\left(\frac{N}{-M}\right)}=\frac{-1}{12 . N} \quad\left(0=\left(\frac{N}{-M}\right)\right)-3(\mathrm{~b})$

This gives $12 \mathrm{~N}=\left(\frac{N}{-M}\right)-3(\mathrm{c})$

Substituting this in equation $-3(\mathrm{~b})$

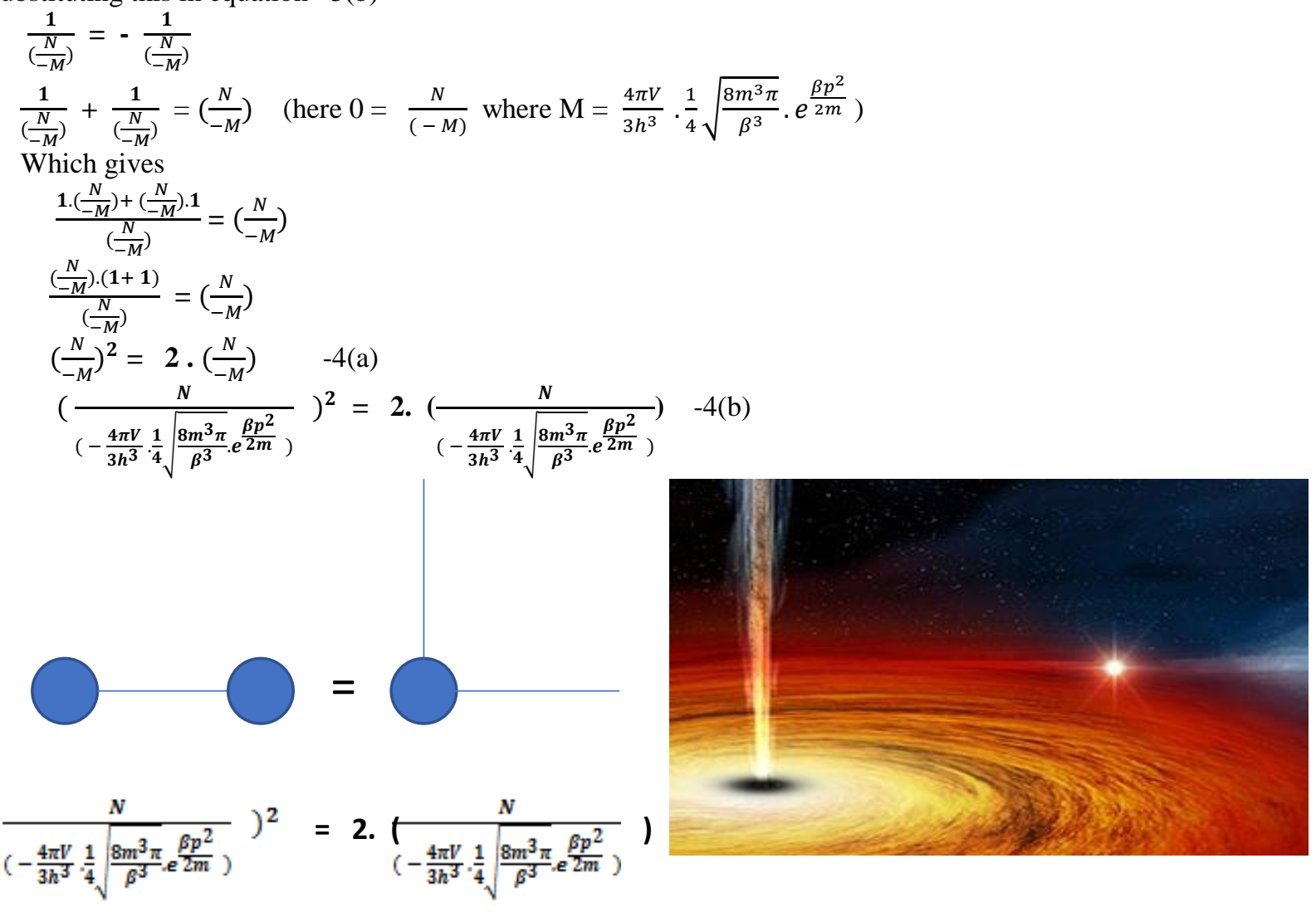

(image: NASA) Fig. 1

For the system of $\mathrm{n}$ class zero particles in eq.-4(a) this gives -

$$
\left(\frac{N}{-M}\right)^{2 n}=2 \mathrm{n} \cdot\left(\frac{N}{-M}\right) \quad-4(\mathrm{c})
$$

This proves The Above Equation Of Space Creation.

The created space is maintained and adjusted by the infinity view ( $\left.\infty=\frac{-M}{N}\right)$ which created by matter and nonintractable particle.

for $\mathrm{n}=2$ in eq-4(c) this gives

Then $\quad \begin{aligned}\left(\frac{N}{-M}\right)^{2 n} & =2 \mathrm{n} \cdot\left(\frac{N}{-M}\right) \quad \text { if } \mathrm{n}=2 \\ \left(\frac{N}{-M}\right)^{4} & =4 \cdot\left(\frac{N}{-M}\right)\end{aligned}$

This can be written as $-\left(N\left(-\frac{3 h^{3}}{4 \pi V} \cdot 4 \sqrt{\frac{\beta^{3}}{8 m^{3} \pi}} \cdot \frac{1}{e^{\frac{\beta p^{2}}{2 m}}}\right)\right)^{4}=4 .\left(\mathrm{N}\left(-\frac{3 h^{3}}{4 \pi V} \cdot 4 \sqrt{\frac{\beta^{3}}{8 m^{3} \pi}} \cdot \frac{1}{e^{\frac{\beta p^{2}}{2 m}}}\right)\right)$

Which gives
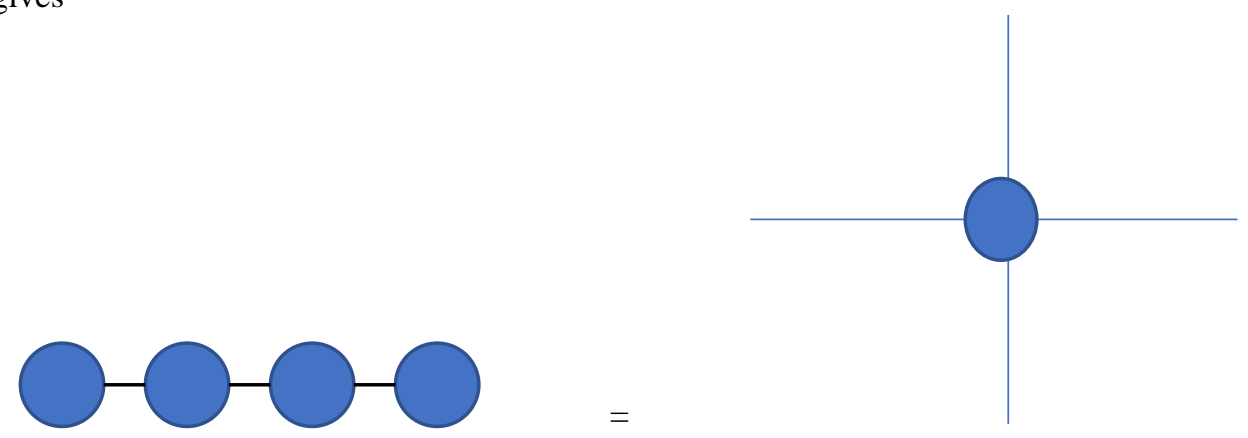

Fig. 2 
The Concept Of Coordinate Axes is First Given By The Mathematician Rene Descartes But In Non-Interactive Mechanics Non-Interactive Particle And Disintegrated Matter is Present Before The Creation Of Coordinate Axes In The Universe And Both Non-Intractable Particle And Disintegrate Matter Creates And Maintain The Coordinate Axes In The Universe. Now We Study The Behavior Of Equation -4 c Which Is $\left(\left(\frac{N}{-M}\right)^{2 n}=\mathbf{2 n} \cdot\left(\frac{N}{-M}\right)\right)$ As We Conducted The Operation Above According To That Zero View is $0=\left(\frac{N}{-M}\right)$ If We Substitute This Condition In Equation $-4(\mathrm{c})$ Then It Transform As $(\mathbf{0})^{\mathbf{2 n}}=$ 2n .(0) The L.H.S Of This Equation Describes The Infinite River Of Quantum Domains Or Class Zero Particles. In The Universe And The R.H.S Of Equation Represents The Space Adjustment Nature .

\section{Condition For Appearance Of Time In The Universe-}

now dividing equation $-4(\mathrm{c})$ by $\left(\frac{N}{-M}\right)$ on both sides-

$$
\frac{\left(\frac{N}{-M}\right)^{2 n}}{\left(\frac{N}{-M}\right)}=\frac{2 n \cdot\left(\frac{N}{-M}\right)}{\left(\frac{N}{-M}\right)}
$$

This can also be written as-

$\left(\frac{N}{-M}\right)^{2 n-1}=2 \mathrm{n}\left(\frac{N}{-M}\right)^{1-1}-\mathbf{5}(\mathrm{a})$

This Can Written As

$\left(\frac{N}{-M}\right)^{\mathbf{2 n - 1}}=\mathbf{2} \mathbf{n}\left(\frac{N}{-M}\right)^{\mathbf{1 + 0 - 1}}$ - Mathematically This Doesn't Violates Above Mentioned Condition

$\left(\frac{N}{-M}\right)^{2 n-1}=2 \mathrm{n}\left(\frac{N}{-M}\right)^{1+\left(\frac{N}{-M}\right)-1}$ As $\left(0=\frac{N}{-M}\right)-(5 b)$

$\left(\frac{N}{-M}\right)^{2 n-1}=2 \mathrm{n}\left(\frac{N}{-M}\right)+\left(\frac{N}{-M}\right)-$

Now If We Look The Behavior Of R.H.S Of Equation - (5c) Then The Mathematical Terms Cross Each Other But We Know That The Non-Intractable Particle Holds The Infinite Expansion Property With Respect TO Constant Disintegrated Matter In Zero View So Even The Mathematical Terms Cross Each Other But The Charge Nature Associated With Them Hold Their Existence With Non-Intractable Particle And Disintegrated Matter So The Elusive Particle $\left(+\left(\frac{N}{-M}\right)-\right)$ Find Its Existence In The existence In The Equation -5(c)

Now taking L.H.S of equation -5(a)

$\left(\frac{N}{-M}\right)^{2 n-1}$,which can be written as $\left(\frac{N}{-M}\right)^{2 n} \cdot\left(\frac{N}{-M}\right)^{-1}$ now figuring this gives-

A

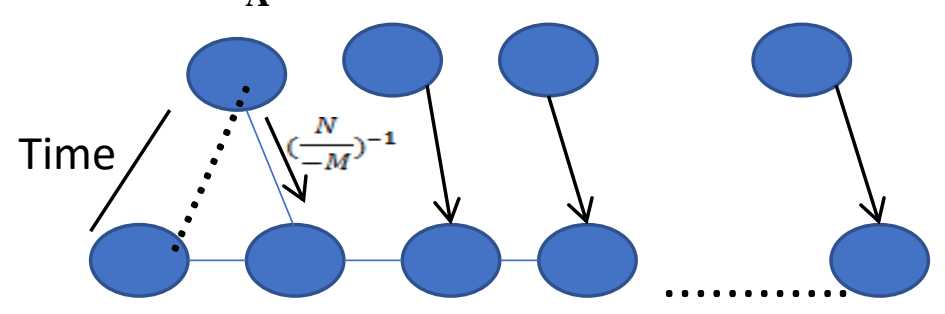

Fig...3

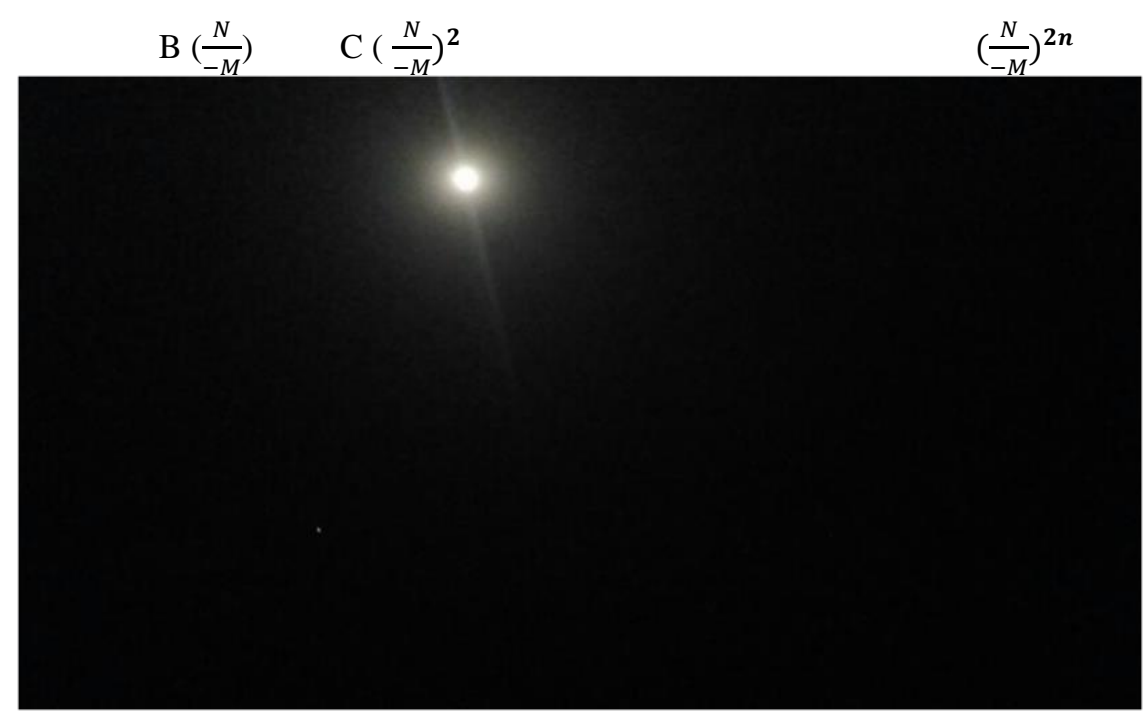

Fig. 4 
The Image Was Taken On 12-04-17 On Between 8:30 PM to 9:30PM In Gadarpur, U.S.Nagar,Uttrakhand, India. Credit: Nishant Kumar Sharma.

The Term $\left(\frac{N}{-M}\right)^{2 n}$ Is Showing The River Of Class Zero Particle And The Term $\left(\frac{N}{-M}\right)^{-\mathbf{1}}$ Is Representing The Strike Of Infinite View With Zero View As We Know $\infty=\frac{-M}{N}$, This Explain How Zero View Holding Field Quanta Strike With The Infinite Universe, Here In Above Figure We See That The Particle A Strike With Particle C The Second Particle Of The River OF class Zero Particles Or We Can Say That the remaining part between state A and state B is ' Time ' Or The Space Which Is Not Occupied By Matter, In Other Words We Can Say This Is The Starting Of Time In An Universe Everyday.

\section{Superiority Of Space Over Time And Origination Of Electric And Magnetic Nature In Universe- Space Is Primary Component Than Time In Universe}

According To Above Figures We Finds That Space Creation Or Linear Expansion Of Not-Intractable Particles Have Its Existence Before The Striking Of Infinity View Particles, As The First Non-Intractable Particle Of River Of Class Zero Particle Is Free From The Condition Of Time This Explains That Space And Time Are To Different Phenomena Of NonIntractable Particles And Matter And Universe And Space Is Primary Component Than Time In Universe.

\section{Electric Nature Is Primary Than The Magnetic Nature In The Universe}

The Linear Expansion Of River Of Class Zero Particles And Their Entering In The Matter Gives Charge Nature To Non Particles And Its Expansion And adjustment Is Happen According To The Condition Mentioned In The Equation 4(c) $\left(\frac{N}{-M}\right)^{2 n}=2 n \cdot\left(\frac{N}{-M}\right)$ Here The R.H.S Component Explains The Space Adjustment Of Non-Intractable Particles Here The Horizontal X- Axis Explains The Electric Nature Or Continuous Flow Non-Intractable Particles And Vertical Y-Axis Explains Magnetic Nature As It Strike Back To Horizontal X-Axis But The Behavior Of Source Of Class Zero Particles Is Horizontal Or We Can Say That Class Zero Particles Continuously Flows In The Horizontal Direction.

This Explains The Electric Nature Is Primary Nature Of Class Zero Particles Than Magnetic Nature And Magnetism Is An Emerged Nature From Electric Nature 'Which Is Same As A Conditions Of Electrodynamics And Experimental Proofs Of Electricity And Magnetism'.

5.The Concept Of Non-Intractable Particles Under Disintegrated Matter (-M) And Beyond Disintegrated Matter (M)

The Concept Of The Particles Under Disintegration Or Beyond Disintegration Is Depend on The Dividing Properties Of Particles Such As Atom is Undivided Unless The Required Energy Is Applied To It To Break It Into Proton, Electron And Neutron And Even These Particles Are Non-breakable And Non Divided Unless The Required Energy Applied To It When The Required Energy Applied To It They Breaks Into Quarks And Than Gluons So The Particle Is Under Disintegrated Or Beyond Disintegrated Matter It Depend On The Energy Applied To The Particle The Applied Energy React With Disintegrated Matter And The Disintegrated. Matter Turns Into Energy and Decide That The Particle Will Be Under Disintegrated Matter Or Not.

So For Particles Under Disintegrated The Applied Energy Should Be Less Then Disintegrated Matter

a. $\mathrm{E}<-\mathrm{M}$

For Particles Beyond Disintegrated Matter The Applied Energy Should Be Greater Than Disintegrated Matter b. $\mathrm{E}>-\mathrm{M}$

Example: The Existence Mass Of Vector bosons W+ And W- is $80.9 \pm 2.0 \mathrm{Gev} / \mathrm{C}^{\wedge} 2$ (Source: CERN 1983)

First The Equation Is Same As The Form $\mathrm{N}=-\mathrm{M} \pm \mathrm{F}$ p.of

So Here Disintegrated Matter $(-\mathrm{M})=80.9$

Formation Mass of Vector Bosons = 2.0

So For Vector Bosons Beyond Disintegrated Matter Applied Energy Should Be Higher Than 80.9 Gev/C^2 .

For Vector Bosons Under Disintegrated Matter Applied Energy Should Be Lower Than 80.9 Gev/C^2 .

This Left A Remarkable Impact On The Condition Of Systems As It Change The Reacting Nature Of The Particles And Even The Communication Between Particles And System. These Systems Under Disintegrated Matter And Beyond Disintegrated Matter Will Be Mentioned Ahead.

According To The Non-Interactive Mechanics-

$$
\begin{aligned}
& N=-\frac{4 \pi V}{3 h^{3}} \cdot \frac{1}{4} \sqrt{\frac{8 m^{3} \pi}{\beta^{3}}} \cdot e^{\frac{\beta p^{2}}{2 m}} \pm \sqrt{N^{2}+2 N \frac{4 \pi V}{3 h^{3}} \cdot \frac{1}{4}\left(\sqrt{\frac{8 m^{3} \pi}{\beta^{3}}}\right) \cdot e^{\frac{\beta p^{2}}{2 m}}+\left(\frac{4 \pi V}{3 h^{3}} \cdot \frac{1}{4}\left(\sqrt{\frac{8 m^{3} \pi}{\beta^{3}}}\right) \cdot e^{\frac{\beta P^{2}}{2 m}}\right)^{2}} \\
& \mathbf{N}=-\mathbf{M} \pm(\mathbf{f} \text { p.o.f }) \quad \text { Here } \mathrm{N} \text { Is Non-Intractable Particle , -M Is Disintegrated Matter And (f p.o.f ) is Process Of } \\
& \text { Formation. }
\end{aligned}
$$


Now According To This Equation Non-Intractable Particles Are Two Types-

1. The Non-Intractable Particles Which Have The Velocity $299792458 \mathrm{~m} / \mathrm{sec}$ (The Velocity Of Light) Are Under Disintegrated Matter (-M) .These Particles Loose Mass With Every Step Of Their Movement And Reach To Stage Where Their Rest Mass Becomes Equals To 0 Such Photon And Graviton.

2. The Non-Intractable Particles Which Have The Velocity $299813275.22 \mathrm{~m} / \mathrm{sec}$ Are Beyond Disintegrated Matter (-M). That Means By The Distribution Of These Particles Could Divide And Joint Together In Almost Limit less numbers. And Even After Divide And Joint In Limitless Range The First Non-Intractable Particle Remains As Same As Before. Such As Non-Intractable Particle N, Class Zero Particles $(\mathrm{N}+\mathrm{M}=0)$.

According To This Distribution Of Non-Intractable Particles Under And Beyond Disintegrated Matter (-M) We will Study The Varies Systems For The Movement of Non-intractable Particles Under And Beyond -M.

6.1 The Varies Quantum Mechanical systems for the Movement of Elementary And Non-Intractable particles -

\section{The N-I particles under Disintegrated Matter( $-\mathrm{M})$}

Now According To The Equation -5 (b) $\left(\frac{N}{-M}\right)^{2 n-1}=\mathbf{2} \mathbf{n}\left(\frac{N}{-M}\right)^{1+\left(\frac{N}{-M}\right)-1}$

Taking R.H.S Of Above Equation Can Be Written As 2 n $\left(\frac{N}{-M}\right)^{\mathbf{1}} \cdot\left(\frac{N}{-M}\right)^{+\left(\frac{N}{-M}\right)-} \cdot\left(\frac{N}{-M}\right)^{\mathbf{1}}$

Figuring The Above Equation Gives Two Conditions-

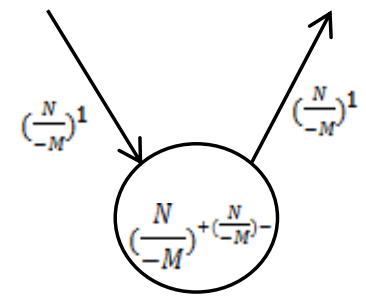

$n$ a.

The figures a. And b. ,Gives the system for the movement of class zero particles whose combined form is

b.

Fig . 5

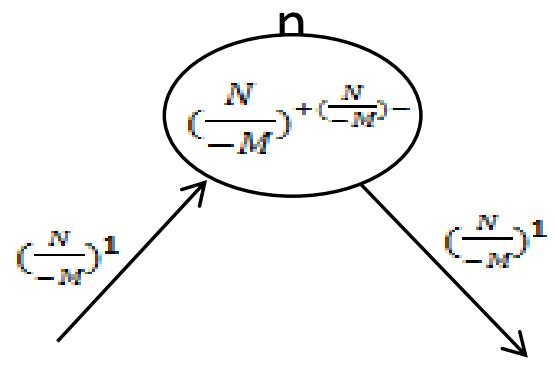

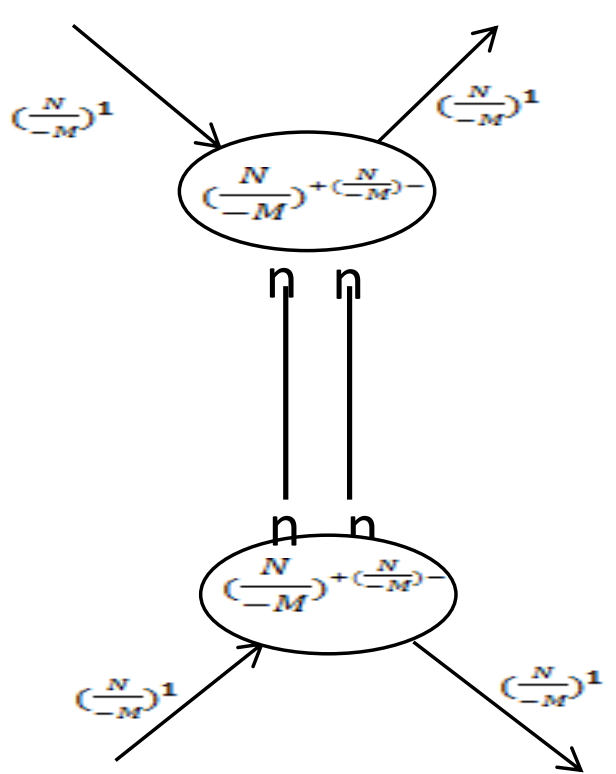

Fig. 6 


\section{RESULTS AND DISCUSSION}

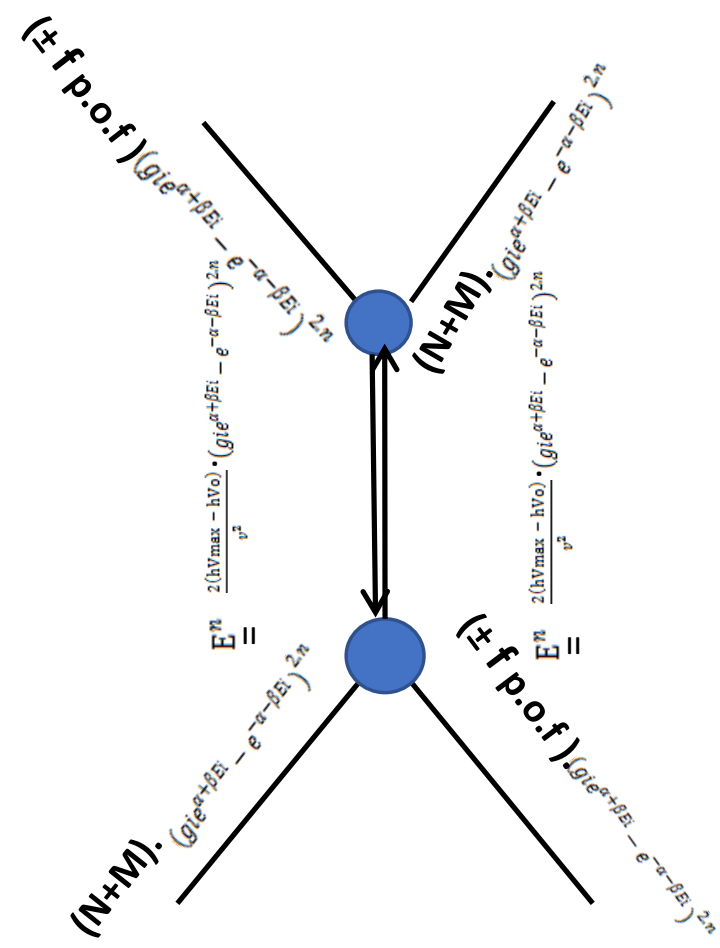

Fig.7 Image- $f$ Kumar Sharma

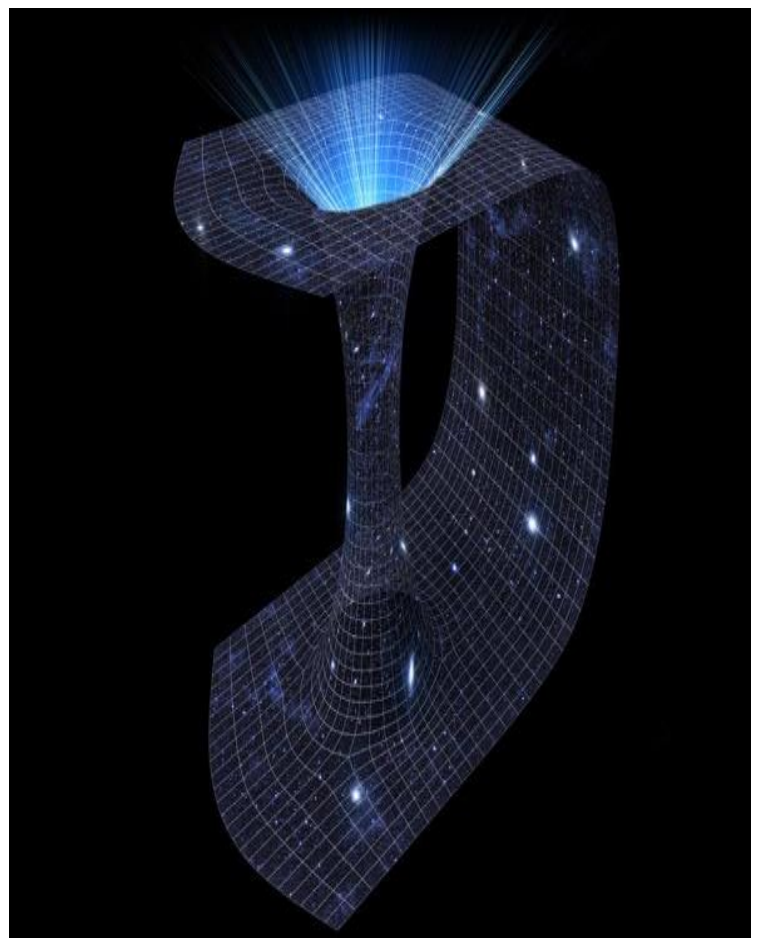

Fig. 8 Image- https://www.sciencenews.org/blog/context/new-einstein-equation-wormholes-quantum-gravity

The upper Image Is According To The Non-Interactive Mechanics The Image Follows The Nishant Effect And The Relations In It Proved Several Eq. Like $1 . \mathrm{E}=1 / 2 \mathrm{hv} 2 . \mathrm{E}=\mathrm{hv} \quad 3 . \mathrm{E}=\mathrm{mc}^{\wedge} 24 . \mathrm{E}^{\wedge} 2=\mathrm{P}^{\wedge} 2 \mathrm{C}^{\wedge} 2+\mathrm{M}^{\wedge} 2 \mathrm{C}^{\wedge} 45 . \mathrm{The}$ Approximation of Velocity Of Light. This Figure Explains The Required Quantum Mechanical And Non-Interactive Mechanical Conditions For The Creation Of Wormholes. 
The lower Image Is Predicted By A. Einstein In His Theory Of Wormholes.

We Have Quantum Teleportation In current Days So Why We Need This Teleportation System The Quantum Teleportation System Follows The Current Modern Physics Models Of Nucleus And Atom Which Says Nucleus Is Composite Of Proton And Neutrons And Electrons Circulate Around Nucleus Which Is Correct But It Doesn't Describe That Protons And Neutrons Are Arranged In Different Cells, As It Mentioned In The Non-Interactive Mechanics.

The Teleportation In Which We Can Dematerialize An Object And Regain It On Other Side Of Space And Time For This The Structure Of Atom And Nucleus Is Mentioned Below According To It Positively Charged Protons Is In Dense Energy Fields Cell , Neutrons Are In Dense Energy Fields Neutral Cell And Electrons Circulate Around It In Spread Energy Field With Respect To Four Curve Geometries.

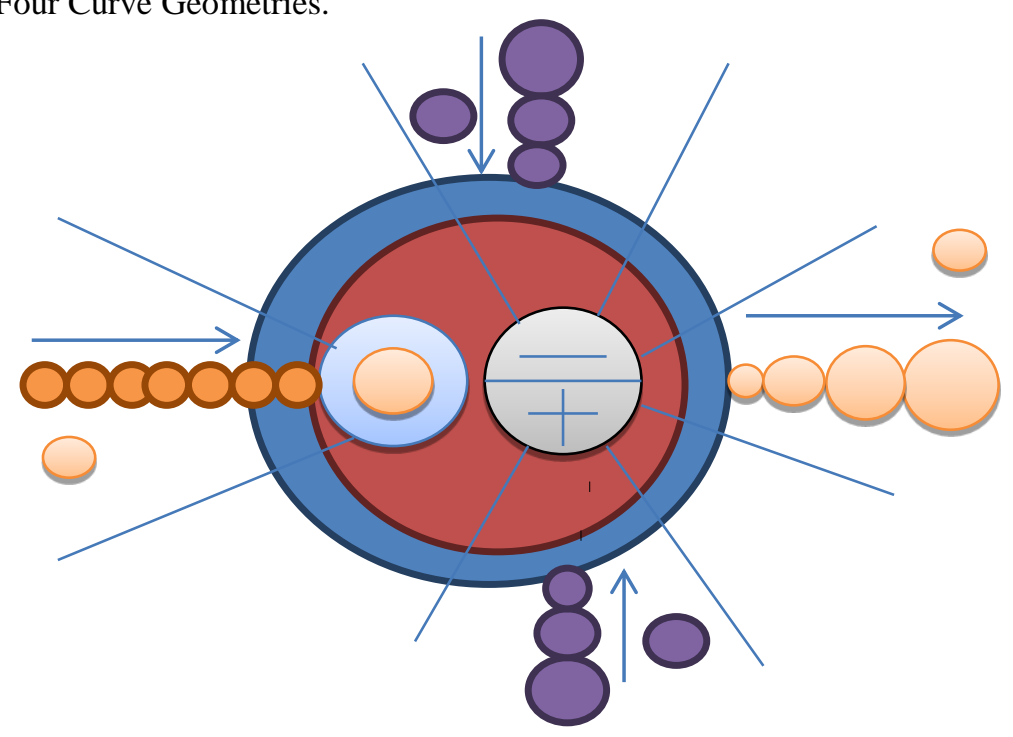

Fig.9

\section{The N-I particles beyond $-\mathrm{M}$}

Now According To The Equation $-5\left(\right.$ b) $\left(\frac{N}{-M}\right)^{2 n-1}=\mathbf{2} \mathbf{n}\left(\frac{N}{-M}\right)^{1+\left(\frac{N}{-M}\right)-1}$

Taking R.H.S Of Above Equation Can Be Written As 2 n $\left(\frac{N}{-M}\right)^{\mathbf{1}} \cdot\left(\frac{N}{-M}\right)^{+\left(\frac{N}{-M}\right)-} \cdot\left(\frac{N}{-M}\right)^{\mathbf{1}}$
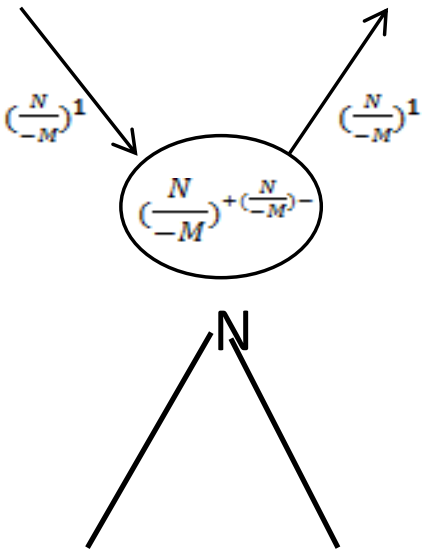

the figures are A.

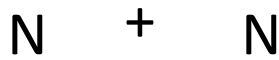

B.

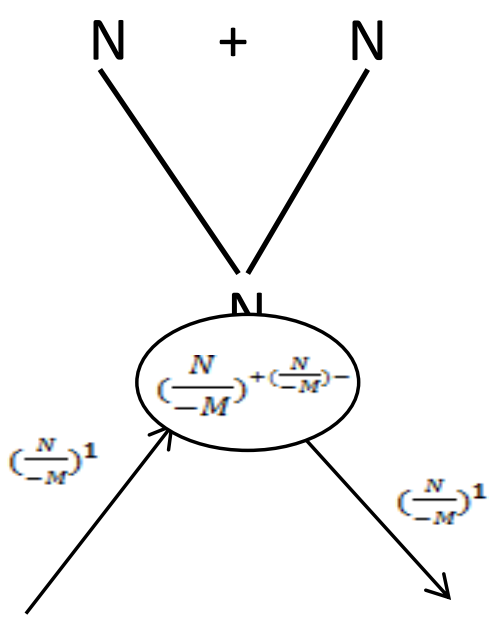

Fig. 10

These are the two states of a same system, now after combining these two figures gives, the system for the movement of class zero particles which are beyond '-M'. 

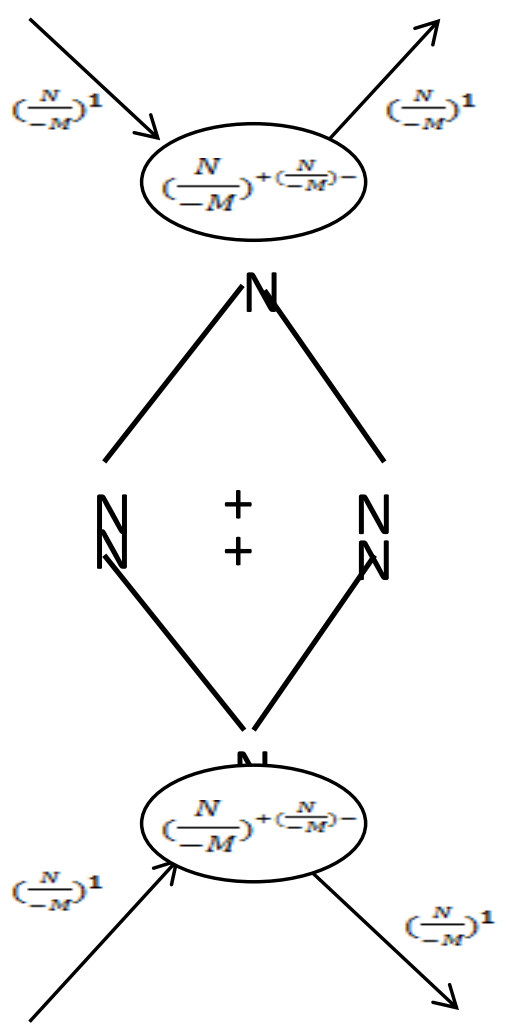

Fig. 11

6.2 Large Scale Systems For The Movement Of Non-Intractable Particles Under Disintegrated Matter (-M) And Beyond Disintegrated Matter (-M)

So Finding The Large Scale Systems Let Multiply Eq.- 5(a) and -5(b)

$\left(\frac{N}{-M}\right)^{2 n-1}=2$ n $\left(\frac{N}{-M}\right)^{1-1}-(5 a)$

$\left(\frac{N}{-M}\right)^{2 n-1}=2 \mathrm{n}\left(\frac{N}{-M}\right)^{+\frac{N}{-M}-}-(5 \mathbf{c})$

$\left(\frac{N}{-M}\right)^{2 n-1} \cdot\left(\frac{N}{-M}\right)^{2 n-1}=2 \mathrm{n}\left(\frac{N}{-M}\right)^{1-1} \cdot 2 \mathrm{n}\left(\frac{N}{-M}\right)^{+\frac{N}{-M}-}$

$\left(\frac{N}{-M}\right)^{4 n-2}=4 n^{2} \cdot\left(\frac{N}{-M}\right)^{1+\left(\frac{N}{-M}\right)-1}$

So figure for L.H.S of equation (6) Can Be Written As

$\left(\frac{N}{-M}\right)^{4 n-2}=\left(\frac{N}{-M}\right)^{4 n} \cdot\left(\frac{N}{-M}\right)^{-1} \cdot\left(\frac{N}{-M}\right)^{-1}$ The Figure For This Condition Is

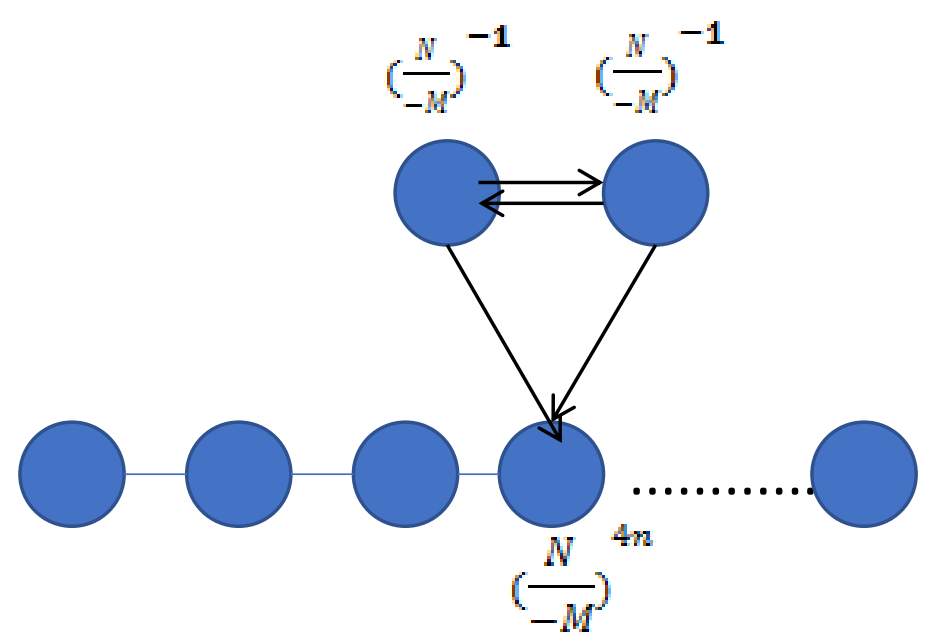

Fig. 12 
Now the R.H.S of equation $-(6)$

$$
\left(0^{4} \text { when } \mathrm{n}=1\right)
$$

$4 n^{2} \cdot\left(\frac{N}{-M}\right)^{1+\left(\frac{N}{-M}\right)-1}=4 n^{2} \cdot\left(\frac{N}{-M}\right)^{1} \cdot\left(\frac{N}{-M}\right)^{+\left(\frac{N}{-M}\right)-} \cdot\left(\frac{N}{-M}\right)^{1}$

The figure for this value is two types according to equation $\quad \mathbf{N}=-\mathbf{M} \pm(\mathbf{f}$ p.o.f $)$

i. $\quad \mathrm{n}$ Under Disintegrated Matter $(-\mathrm{M})$

ii. $\quad \mathrm{n}$ Beyond Disintegrated Matter (-M)

i. For n under Disintegrated Matter $(-\mathrm{M})$

the figure for given condition is -

a.

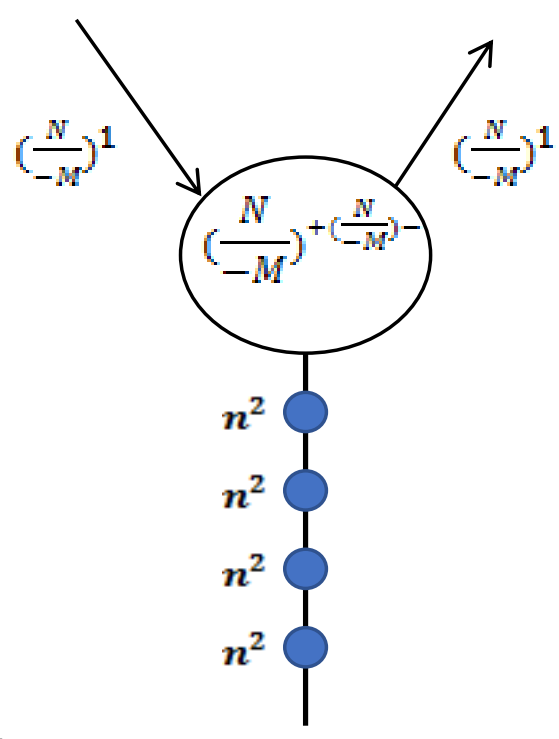

b.

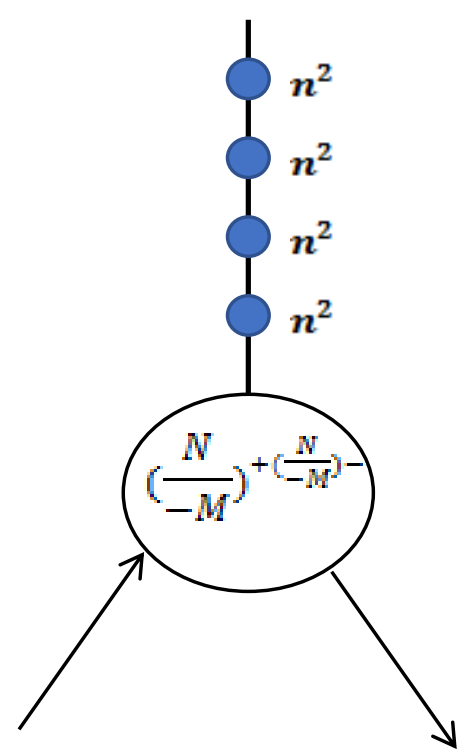

Fig. 13

combined form of given figure is by joining a. And b. We get -

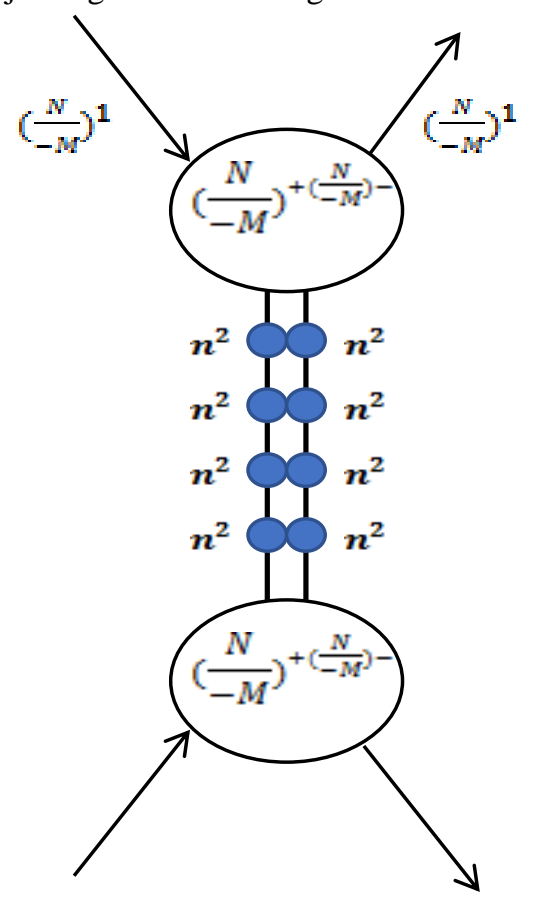

Fig. 14

ii. n Beyond Disintegrated Matter $(-M)-$ for this form $n=N$ so figure is 

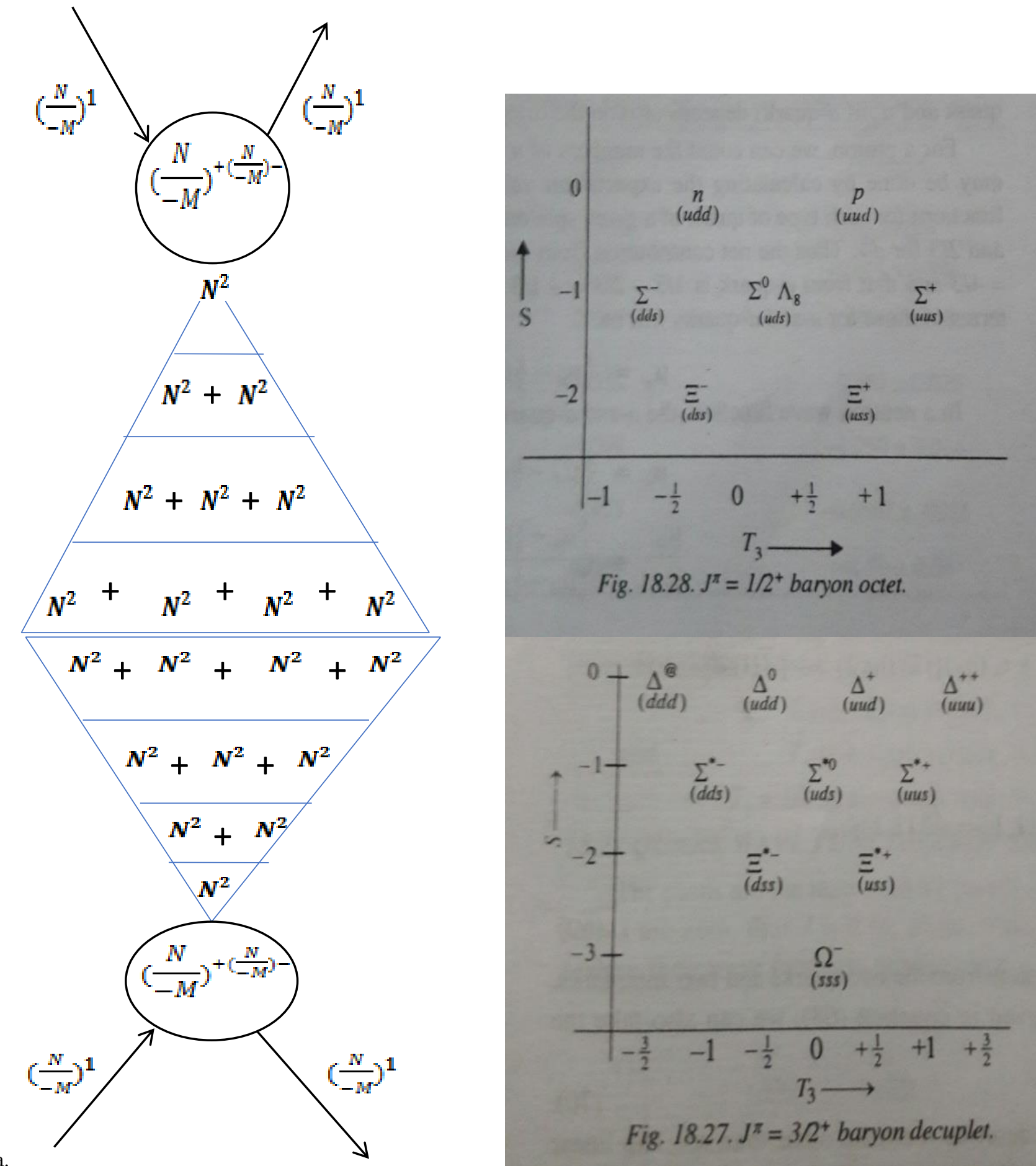

Fig. 18.28. $J^{\pi}=1 / 2^{+}$baryon octet.

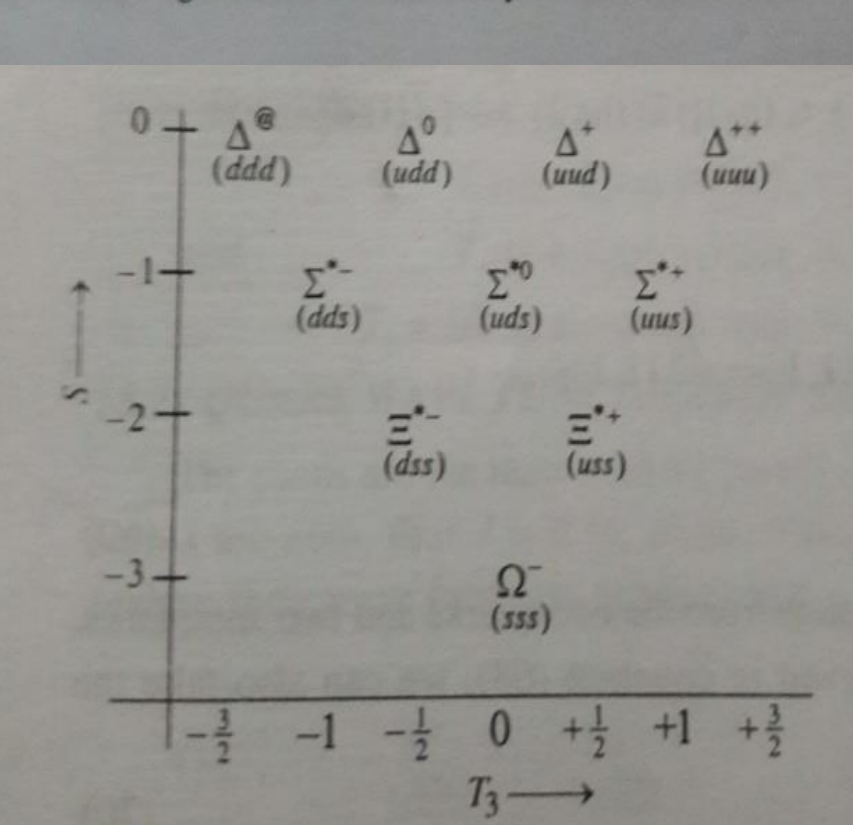

Fig. 18.27. $J^{\pi}=3 / 2^{+}$baryon decuplet.

Credit : D.C.Tayal, Nuclear Physics

Fig. 15

We Have Clear Set Of Downward Direction, But For Upward Direction We Don't Have 2 Particles Which Are NonIntractable Particle And Graviton, Non-Interactable Particle Will Show Positive Nature In Matter Field While Graviton Will Show Nutral Nature In Matter Field.

The Combined Figure Of a. And b. is 


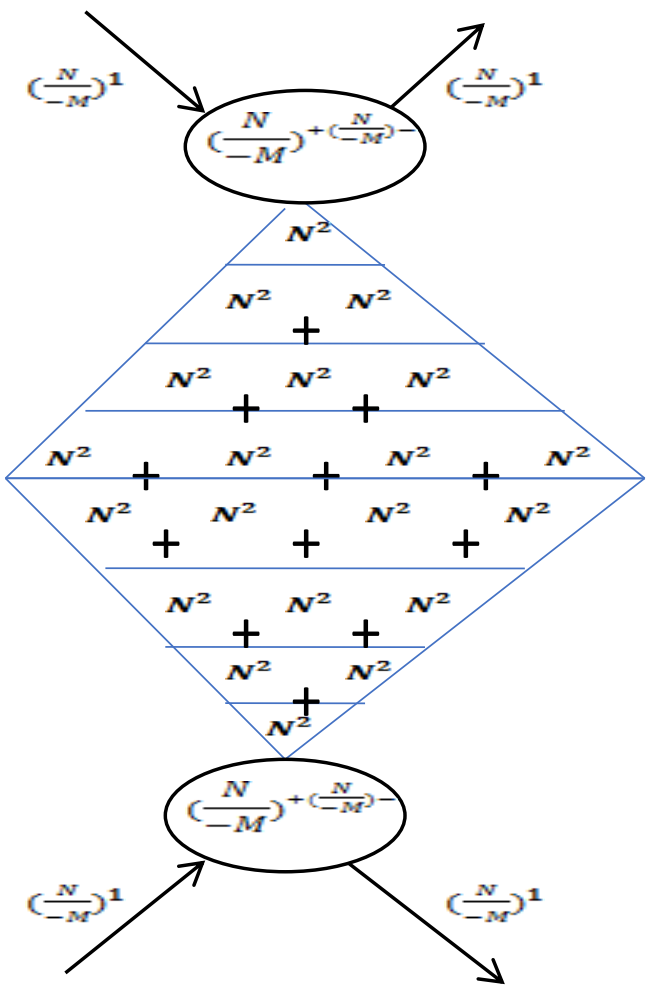

c.

Fig. 16

At Large Scale Universe Non-intractable Particles Move Through Above Mentioned Figure

7.1Concept of Approximately Stable System (Planets, Stars) -

According To The Non- Intr. Mechanics The Structure Of Atom And Nucleus Have Spread And Energy Fields And These Fields Are Lead By Four Curve Space Geometries, When These Systems Comes Under These Curved Space Geometries ,Space And Time Bend Their Structure And This Occurs To The Starting Of Approximately Stable Systems Such As Planets And Stars.

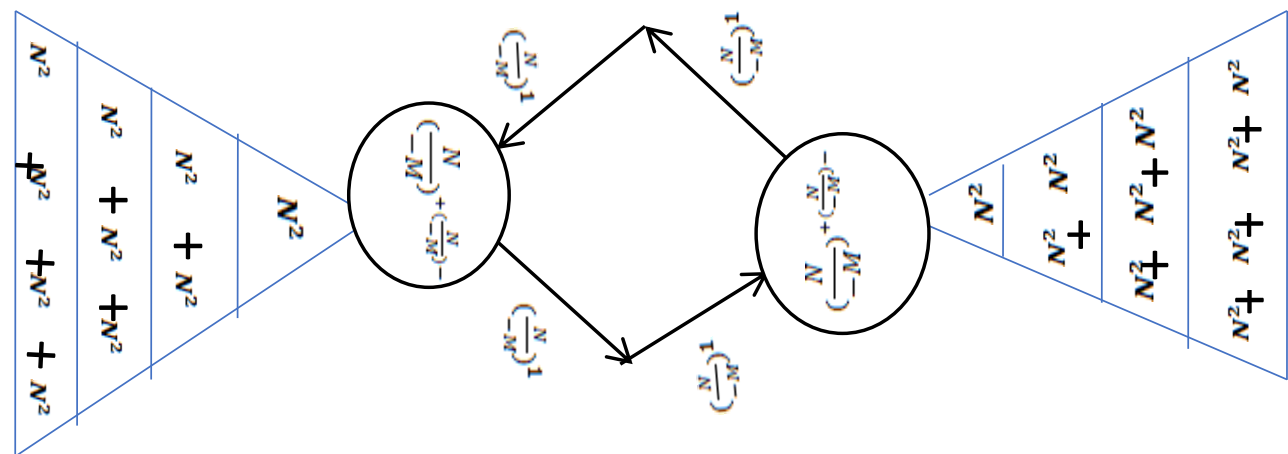

Fig. 17

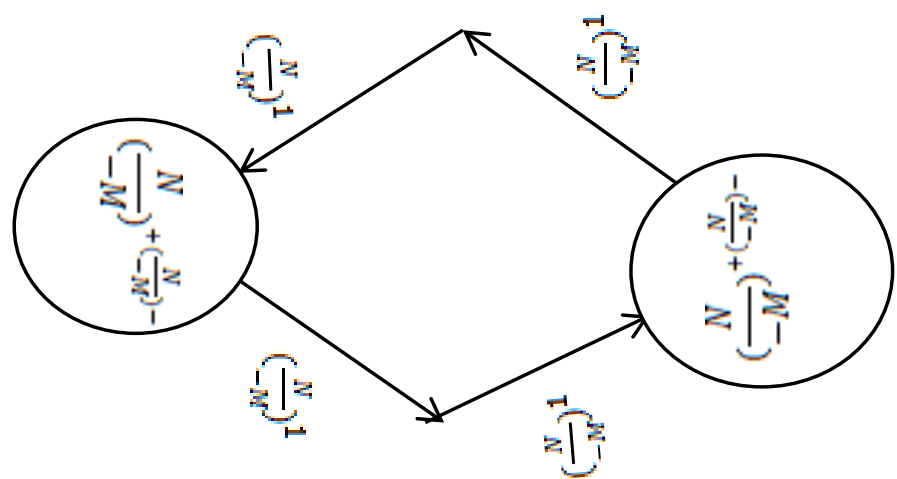

Fig. 18 
7.2 Aatmamoul The Quantum Mechanical systems for the Movement of Elementary And Non-Intractable particlesAccording To Above Mentioned Figures The System Is Available For The Movement Of Class Zero Particles

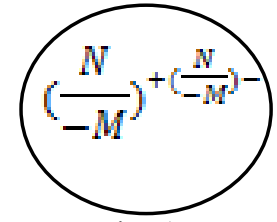

Fig. 19

This Is The System For Movement For The Movement Of Class Zero Particles The System Is Name According To My Opinion Is Aatmamoul. According To Above Figure Equation Is $\left(\frac{N}{-M}\right)^{+\left(\frac{N}{-M}\right)-}$ This Explain That Expansion Of NonIntractable Particles Happens With Respect To Two Disintegrated Matters One Gives Charge Nature And Other Gives Mass To Non-Intractable Particles. Which Is Same As The Figure Of Atom And Nucleus According To Non-Interactive Mechanics.

8. Concept Of Adjustment of Quantum Domains- Para-Kans and $\left(\frac{N}{-M}\right)^{+\left(\frac{N}{-M}\right)-}$ -

The Aatmamoul $\left(\frac{N}{-M}\right)^{+\left(\frac{N}{-M}\right)-}$ is defining the limitless extension properties as Mentioned In Above Equation. In Our Universe We See The Limitless Extension Of Particles And Matter And Even That We Observe That Matter And Particles Are Properly Arranged. Here The Need Of Particles Which have Infinite Divide And Infinite Attachment Nature Is Required These particles are 'PARA-KANS', the particles posses infinite extension property with the unique nature to hold infinite PARA-KANS, These Particles Balance The Extension And Contraction Nature Of Universe. Now We Will Study These Two Types Of Para-Kans Mathematically And According To Non-Interactive Mechanically.

1. Para-Kans Having Infinite Divide Property

This system provide the infinite divide properties is hold by Para-Kans, mathematically $\frac{N}{-M}=\frac{N}{-M}+\left(\frac{N}{-M}\right)-\left(\frac{N}{-M}\right)+\left(\frac{N}{-M}\right)-\left(\frac{N}{-M}\right) \ldots \ldots$

This state can be define as According To The Figure ,Para-Kans for infinite divide property-

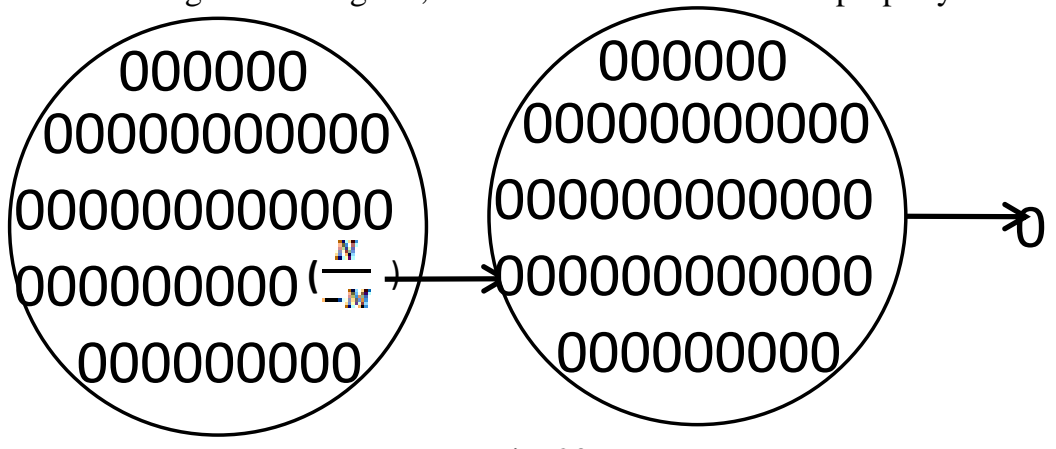

Fig. 20

$\mathrm{f}\left(\frac{N}{-M}\right)=\frac{N}{-M}+\left(\frac{N}{-M}\right)-\left(\frac{N}{-M}\right)+\left(\frac{N}{-M}\right)-\left(\frac{N}{-M}\right) \ldots . . \quad($ so almost infinite zeroes or $\infty(0))$

$\mathrm{f}\left(\frac{N}{-M}\right)=\frac{N}{-M}+\left(\left(\frac{N}{-M}\right)+\left(\frac{N}{-M}\right) \ldots ..\right)+\left(-\left(\frac{N}{-M}\right)-\left(\frac{N}{-M}\right) \ldots.\right)$

here the first class zero $\left(\frac{N}{-M}\right)$ particles is free from sign variations, as then arranging the remaining sign in according to their sign.

$\left.\mathbf{f}\left(\frac{N}{-M}\right)={ }^{6} \frac{\boldsymbol{N}}{-\boldsymbol{M}} \cdot+\left(\sum_{\frac{N}{-M}}^{\infty}{ }^{\left(\frac{N}{-M}\right.}\right)\left(+\boldsymbol{n} \boldsymbol{f}\left(\frac{N}{-M}\right)\right)+\sum_{\frac{N}{-M}}^{\infty}\left(\frac{N}{-M}\right)\left(-\boldsymbol{n} \boldsymbol{f}\left(\frac{N}{-M}\right)\right)\right) \quad$-equation -(7a)

The equation $-(7 a)$ has two parts-

1. The First Class Zero Particle ' $\frac{N}{-M}$ ' , the reason about it's prime nature is it's away from sign variation $(+,-)$, and posses the infinite extension property.

2. Then the sign varying sets $(+,-)$ these are as equal in quality but opposite in nature and the expansion rate of every Para-kan is $\left.\left(\sum_{\frac{N}{-M}}^{\infty} \frac{N}{-M}\right) f\left(\frac{N}{-M}\right)\right)$ similar as previous.

2. Para-Kans Having Infinite attachment property second state of Para-kans is infinite attachment property if the expansion of para-kans Having In Universe As Mentioned In Above Equation $=7$ (a) Then

$\left(\frac{N}{-M}\right)=\ldots \ldots \ldots . .\left(\frac{N}{-M}\right)+-\left(\frac{N}{-M}\right)+-\left(\frac{N}{-M}\right) \ldots \ldots \ldots$

The figure for the above equation is 

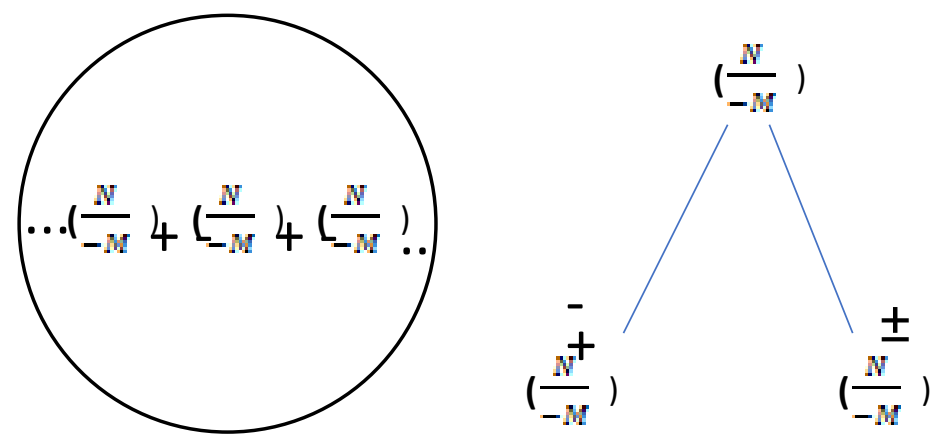

Fig. 21

Mathematically-

$\mathrm{f}\left(\frac{N}{-M}\right)=\ldots . . \frac{N}{-M} \mathrm{~F}\left(\frac{N}{-M}\right) \pm\left(\frac{N}{-M}\right) \ldots$.

$\mathrm{f}\left(\frac{N}{-M}\right)=\ldots \ldots . \sum_{\mathrm{f}\left(\frac{N}{-M}\right)}^{\infty(\mathrm{F})}\left(n f\left(\mathrm{~F}\left(\frac{N}{-M}\right)\right)\right) \mathrm{\mp}\left(\frac{N}{-M}\right) \pm \sum_{ \pm\left(\frac{N}{-M}\right)}^{\infty\left( \pm\left(\frac{N}{-M}\right)\right)}\left(n f\left( \pm\left(\frac{N}{-M}\right)\right) \ldots . .-7(\mathrm{~b})\right.$

Both infinitely divide and infinitely addition nature is hold by Para-kans which makes Universe Expansion And Contraction Rate stable. Where Infinite Divide For Expansion Of Universe And Infinite Attachment For Contraction Of Universe.

\section{Self Moving Conscious Particles}

"Consciousness Is The Procedure Which Conducted By The Exchange Of Class zero Particles In Varies Types Of Systems Which Are Available For Both N-I Particles Under Disintegrated Matter( -M) And Beyond Disintegrated Matter ( -M) And The Exchange Is Done Through The Medium Called Aatmamoul.

In Above Given Figures The Terms $\left(\frac{N}{-M}\right)^{1},\left(\frac{N}{-M}\right)^{+\left(\frac{N}{-M}\right)-}$ And $+\left(\frac{N}{-M}\right)-$ Are The Particles Which Arise From The Self Interactions From The River Of Class Zero Particles In Universe, These Particles Are Arise For Completing The Process Of Creation, This Self Arise Interactions Are Known As 'Consciousness' Which Start For The Creation Of Universe And To Hold And Maintain Universe .

Here The Terms Which Are Provided Which Makes A System Conscious Have Mathematical Relations Which Are-

1. $\left(\frac{N}{-M}\right)^{1}=\left(\mathrm{N} /\left(-\frac{4 \pi V}{3 h^{3}} \cdot \frac{1}{4} \sqrt{\frac{8 m^{3} \pi}{\beta^{3}}} \cdot e^{\frac{\beta p^{2}}{2 m}}\right)\right)^{1}$

This is the exchange quantity in the different systems which are mentioned above, this exchange quantity is one of the primary quantity which play a important role to create a system conscious Or we Can Say This Is Starting Of Consciousness In Systems .

2. $\left(\left(\frac{N}{-M}\right)\right)^{+\left(\frac{N}{-M}\right)-}=\left(\mathrm{N} /\left(-\frac{4 \pi V}{3 h^{3}} \cdot \frac{1}{4} \sqrt{\frac{8 m^{3} \pi}{\beta^{3}}} \cdot e^{\frac{\beta p^{2}}{2 m}}\right)\right.$. $+\left(N /\left(-\frac{4 \pi V}{3 h^{3}} \cdot \frac{1}{4} \sqrt{\frac{8 m^{3} \pi}{\beta^{3}} \cdot e^{\frac{\beta p^{2}}{2 m}}}\right)-\right.$

These Are The Aatmamoul Which Gives Medium For The Movement Of Class Zero Particles

So The term $\left(\left(\frac{N}{-M}\right)\right)^{+\left(\frac{N}{-M}\right)-}$ is Necessary component To Generate consciousness In Systems.

3. $+\left(\frac{N}{-M}\right)-=+\left(\mathrm{N} /\left(-\frac{4 \pi V}{3 h^{3}} \cdot \frac{1}{4} \sqrt{\frac{8 m^{3} \pi}{\beta^{3}}} \cdot e^{\frac{\beta p^{2}}{2 m}}\right)-\right.$

This term can be assume as the work house of consciousness which maintains consciousness stable in the both infinite and zero views.

These Three are the primary relations for creating consciousness in different systems and mediums.

10.Gravitation The Emergent Phenomena From Non-Intractable Particles-

10.1 How Newton To Einstein We Are On The Correct Path Of Gravitation And Why We Need An Extension To Understand Gravitation Correctly

According To Newton-

Now If We Have $\mathrm{m} 1=\mathrm{m} 2$ So

$$
\mathrm{F}=\mathrm{G} \frac{m 1 m 2}{(\boldsymbol{r})^{2}}
$$

$$
\mathrm{F}=\mathrm{G} \frac{\boldsymbol{M}^{2}}{(\boldsymbol{r})^{2}}
$$

Now According To Non-Interactive Mechanics $\mathrm{N}=\boldsymbol{M}^{2}$ This Condition Satisfies The Relativistic Condition $E^{2}=$ $P^{2} C^{2}+M^{2} C^{4}$, So We Have Above Equation In Form

$$
\mathrm{F}=\mathrm{G} \frac{N}{(\boldsymbol{r})^{2}}(t \square \text { is relation satisfy all } t \square \text { ree law of motion })
$$


So We Can See That A Non-Intractable Particles Is Present With Force Of Attraction Or Repulsion Between Two Bodies Having Equal Mass And It Propagate With Universal Gravitational Constant, As We Know That If Non-Intractable Particle Is Present In Matter Field Than It Has Velocity Faster Than Light (According To Non-Interactive Mechanics 20817.22 m/sec Faster Than Current Velocity Of Light) This Means No Other Particle Can Replace It.

This Describe Existence Of A Universal Constant Or Cosmological Constant. Here Newton And Einstein Proved Each Other Right.

Now We Have Problem Of Sir Edwin Hubble That Universe Is Expanding Then How We Have Cosmological Constant , Then According To Non-Interactive Mechanics N = -M Or Non-Intractable Has The Property To Disintegrate Matter Continuously

As the Not-Intractable Particle Have the Infinite Expansion Properties According To The Equation

$$
\begin{aligned}
N & =-M+0 \\
N & =-M+N+M^{\prime} \\
N & =\left(-M+M^{\prime}\right)+N \\
& =\left(-M+M^{\prime}\right)-M^{\prime \prime} \pm(f \text { p.o.f }) \quad \text { as }\left(N=-M^{\prime \prime} \pm\right. \text { (f p.o.f) } \\
& =\left(-M+M^{\prime}-M^{\prime \prime}\right) \pm(f \text { p.o.f }) \\
& =\left(-M+M^{\prime}-M^{\prime \prime}\right)+0 \\
& \left.=\left(-M+M^{\prime}-M^{\prime \prime}\right)+N+M^{\prime}\right) \text { 'so As More }-(Y)
\end{aligned}
$$

Applying Condition -(X) In Above Equation We Have

$$
\mathrm{F}=\mathrm{G} \frac{-M+0}{(\boldsymbol{r})^{2}}
$$

And Then Applying Equation $-(\mathrm{Y})$

$$
\mathrm{F}=\mathrm{G} \frac{\left(-M+M^{\prime}-M^{\prime \prime}\right)+N+M^{\prime \prime \prime} \text { so As More }}{(\boldsymbol{r})^{2}}
$$

Or We Can Only Write $\quad \mathrm{F}=\mathrm{G} \frac{N}{(\boldsymbol{r})^{2}} \quad$ According To Condition $-(\mathrm{Y})$

This Describes Due To Non-Intractable Particle's Continuous Matter Emitting Property Universe Is Continuously Expanding But Even At The Same Time It Has A Cosmological Constant Which Is The Non-Intractable Particle.

This Describes The Study Of Sir Newton, Dr. Einstein And Sir Edwin Hubble Was Right

From This Stage We Have A New Problem As Disintegrated Matter Strike And Makes The Relations

$\mathrm{N}+\mathrm{M}= \pm \sqrt{N^{2}+2 N M+(M)^{2}}$

$\mathrm{N}=-\mathrm{M} \pm \sqrt{N^{2}+2 N M+(M)^{2}}$

This Describe The Current Findings Are Correct But Disintegrated Matter Expand The Field Of Gravitation Due To This More Study Is Required To Understand Field Behavior Of Gravitation.

Gravitation Has A Long Story In The Branch Of Physics The Concept Of Gravitation Arise From Isaac Newton According To Newton The 'Attraction And Repulsion Force Between Two Bodies Is Directly Proportional To The Multiplication Of Their Masses And Inversely Proportional To The Square Of The Distance Between Them. Mathematically

$$
\mathrm{F}=\mathrm{G} \frac{m 1 m 2}{(\boldsymbol{r})^{2}}
$$

here $\mathrm{F}$ is The Force Of Attraction Or Repulsion, $\mathrm{G}$ Is Universal Constant $\mathrm{m} 1$,m2 Are Masses Of Two Bodies And $\mathrm{r}$ Is Distance The Mass Bodies.

But In Non-Interactive Mechanics We Have The Equation N = -M And This Relation When Comes In The Form Of N+M $=0$ Satisfies The Relativistic Relation $(\boldsymbol{E})^{2}=(\boldsymbol{P})^{2}(\boldsymbol{C})^{2}+(\boldsymbol{M})^{2}(\boldsymbol{C})^{4}$ The Relation N = -M Shows That Disintegrated Matter-M Is Emergent From The Non-Intractable Particle As No Material Object Can Go Higher Than The Velocity Of Light But As We Proved In Non-Interactive Mechanics That Non-Intractable Particles Moves Faster Than Light, This Describes That Gravity And It's Field Quanta Graviton Can't Be Detected According To The Law Of Newton, This Law Explain The Behavior Of Gravitation When It Is Present In The Matter Field.

In 1915 A. Einstein Present The General Theory Of Relativity And Describe The Field Equation

$$
\begin{aligned}
\mathrm{R} \mathrm{uv}-1 / 2 \mathrm{R} \operatorname{guv}+\Delta \operatorname{guv} & =\frac{8 \Pi G}{(C)^{4}} \mathrm{~T} \mathrm{uv} \\
\text { And } \quad \mathrm{R} \mathrm{uv} & =0
\end{aligned}
$$

Which Is Same As N+M=0 Where M Is $\frac{4 \pi V}{3 h^{3}} \cdot \frac{1}{4} \sqrt{\frac{8 m^{3} \pi}{\beta^{3}}} \cdot e^{\frac{\beta p^{2}}{2 m}}$ 
This Explains The Curved Geometry Of Space And Time, But This Falls On The Velocity Of Light ,As This Equation Is Applicable To The Range When Light Have The Highest Velocity In The Field But According To Non-Interactive Mechanics Non-Interactive Particles Have The Velocity $299813275.22 \mathrm{~m} / \mathrm{sec}$ And The Current Velocity Of Light Is $299792458 \mathrm{~m} / \mathrm{sec}$ This Suggest That Non-Interactive Particles Are 20817.22m/sec Faster Than The Velocity Of Light , And Above Mentioned Equation Is Correct When Space And Time Is Present In Universe Without Them This Equation Doesn't Hold Its Condition And We Established Equations Which Describes That Space And Time Are Two Created Phenomena Of Disintegrated Matter And Non-Intractable Particles Where Space Is Primary Component Than Time, This Also Explains The Existence Of Non-intractable Particle Before The Origination Of Space And Time In Universe So We Can't Understand Gravitation Just By The Properties Of Space And Time ,Also If The Non-Interactive Particles Are Present In The Field Than Above Mentioned Equation Violate From It's Conditions. This Equation Correctly Describe The Curved Space Time Geometry Which Is Actually Correct But When It Comes The Behavior Of Gravity And Graviton Than If We Could Observe Gravity In Daily Life Than why Can't We Find Graviton In Laboratory? The Reason Is That The Nature Of Gravity Is Not Clearly Explained, We Have Understand Some Properties Of Gravity, But The Total Description Is Yet To Be Found To Understand This.

We Have Few Questions-

1. What is Gravitation Really About?

2. How It Originate?

3. How Much Type Of Gravitation Is Present In Universe?

4. What Is The Field Equation That Could Describe Gravitation?

10.2 What Is Gravitation And How It Originate-

The Famous Apple Experiment Of Newton Is Very Important To Understand Gravitation And Also Einstein's Mass Object Experiment That If We Drop A Mass Object In The Fabric Of Space Time Than Another Objects Circulate Around It. The Condition Of Space Creation Gives The Equation And Figure

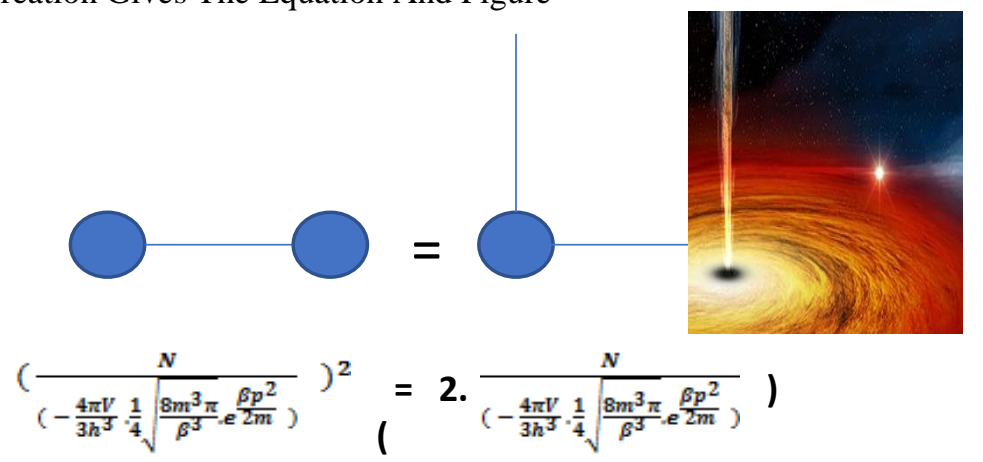

Fig. 22 (image: NASA)

For the system of $\mathrm{n}$ class zero particles

$$
\begin{aligned}
\left(\frac{N}{-M}\right)^{2 n} & =2 \mathrm{n} \cdot\left(\frac{N}{-M}\right) \\
\left(\frac{N}{\left(-\frac{4 \pi V}{3 h^{3}} \cdot \frac{1}{4} \sqrt{\frac{8 m^{3} \pi}{\beta^{3}}} \cdot e^{\frac{\beta p^{2}}{2 m}}\right.}\right)^{2 n} & =2 \mathrm{n} \cdot\left(\frac{N(\mathrm{c})}{\left(-\frac{4 \pi V}{3 h^{3}} \cdot \frac{1}{4} \sqrt{\frac{8 m^{3} \pi}{\beta^{3}}} \cdot e^{\frac{\beta p^{2}}{2 m}}\right)}\right) .
\end{aligned}
$$

https://youtu.be/jfQt-k_ueeQ

This is the live example of Non intractable particle

'The Force Which Applied By Class Zero Particles To Regain Their Primary State Is Gravitational Force'. The L.H.S Of Above Figure Explains The Infinite River Of Class Zero Particles In Universe And R.H.S Explain Their Adjustment In Universe Which Creates Space, The Condition Of Space Creation Is Proved Above. According To This Non-Interactive Particles Are Adjusted In Space In Two ways First Horizontally (X-Axis) And Second Vertically (Y-Axis) The Nature Of Class Zero Particles River Is Horizontal In Universe Due To This The Particles Which Moves Horizontally In Space Adjustment Moves Continuously But The Particles Which Are Adjusted Vertically Repulse To Gain Their Primary Condition And Strike Back To Horizontally Moving Particles, But The Field Quantas Moves Continuously So They Doesn't Attained Their Primary Condition Due To This The Class Zero Particles Disintegrates Matter Continuously And Moves In It, This Moving Force Is Gravitation.

10.3 Types Of Gravitation And Gravitational Waves

Now We Have The Question How Much Type Of Gravitation Is Present In Universe This Will Also Explain That Why We Doesn’t Find Graviton Till Current Days. 
The Answer Is Two Types Of Gravitation We Have In The Universe 1. Expanding Gravitation That Expand Space And Time And Disintegrate Matter And Non-Intractable Particles 2. Contracting Gravitation That Repulse And Contract Space And Time And Disintegrated Matter And Non-intractable Particles.

\section{Expanding Gravitation That Expand Space And Time And Disintegrate Matter And Non-Intractable Particles} In Laboratories We Only Measure Expanding Properties Of Gravitation And It Propagate With Velocity OF Light And If Non-Intractable Particle Is Present Than 20817.22m/sec Faster Than The Velocity Of Light. Due To This Gravitational Force Becomes So Weak To Be Detected In Laboratories AS The Equation OF Einstein Only Explains The Expanding Nature Of Gravitation So We Only Measured The Expanding Force Of Gravitation In Ligo.

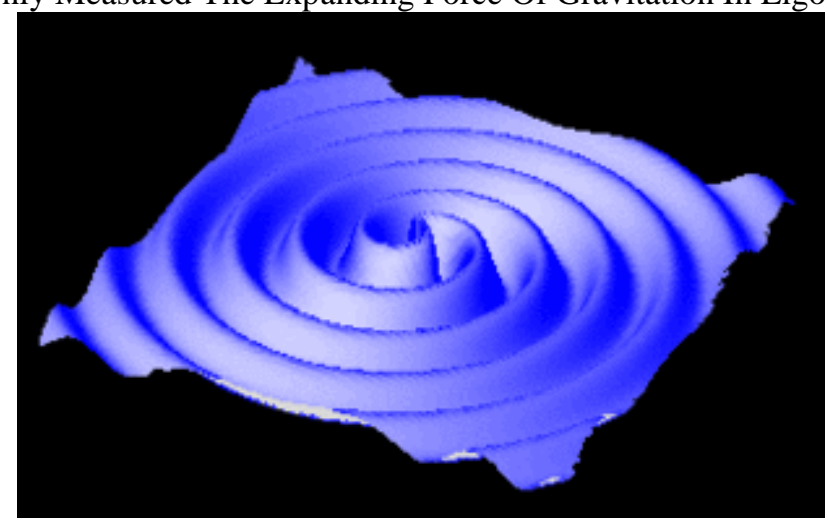

Fig. 23

AS This Figure The Gravitational Waves Expand space By Their Movement. This Explains The Expanding Nature Of Gravitation

2. Contracting Gravitation That Repulse and Contract Space and Time and Disintegrated Matter and Nonintractable Particles, The Test About Contracting Gravitation That Could Be Or May Be Already detected In Ligo Now We Study The Contraction Nature Of Gravitation According To That Space, Time , Disintegrated Matter And NonIntractable Particles Contract By The Force Of Gravitation.

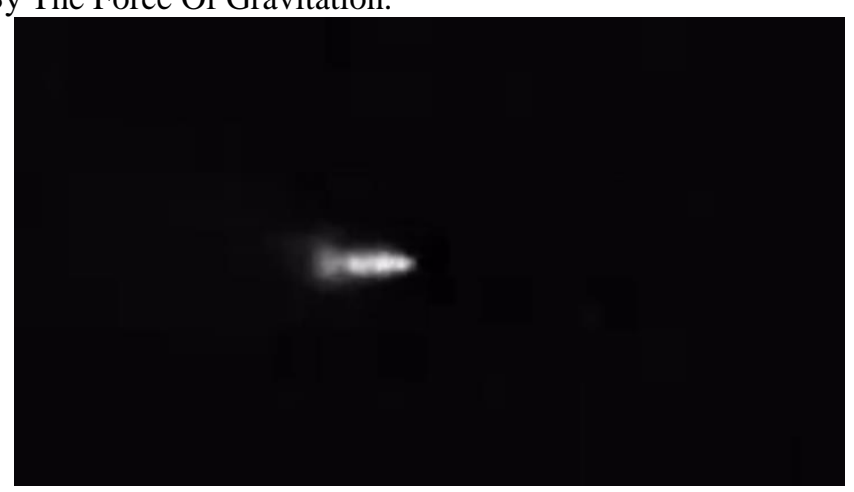

Fig.24 Image: Hubble Space Telescope

https://youtu.be/jfQt-k_ueeQ

As Arrival Of This Type Of Gravitation Is Intend To Regain The Primary Position Of Class Zero Particles, This Type Of Gravitation Follows Ramanujan Entering As Mentioned In Non-Interactive Mechanics According To That 12 N = -1, 12 Non-Intractable Particles Follow Positive Curve Geometry While One Non-Intractable Particle Follow Negative Curve Geometry To Enter In The Matter Field. In Current Days Laboratories We Can Only Measure Positive Curve Geometry So 12 Non-Intractable Particles Or 12 Black Holes Could Be Detected In Ligo Or We Know That NonIntractable Particles Come In Pair So 6 Sets Of Non-Intractable Particles or 6 Sets Of Black Hole Could Be Detected In The Process To Measure The Gravitational Waves In Ligo.

Now The Gravitational Waves Are Two Type 1.Which Expand Space, Time, Disintegrated Matter And Non-intractable Particles In Universe, 2. Which Contract Space, Time, Disintegrated Matter And Non-intractable Particles In Universe

\subsection{The Equations To Describe The Behavior Of Gravitation-}

For Explaining The Behavior Of Two Types Gravitational Waves We Use The Equations Of Para-Kans Which Have The Ability To Expand And Contract Universe

First The Equation Of Expanding Nature Of Gravitation 


$$
\left.\left.\mathrm{F}\left(\frac{N}{-M}\right) \text { Attachment }=\ldots \ldots . \sum_{\mathrm{F}\left(\frac{N}{-M}\right)}^{\infty(\mathrm{T})}+\frac{N}{N}\right)\right)\left(n f\left(\mathrm{~F}\left(\frac{N}{-M}\right)\right)\right) \quad \mathrm{F}\left(\frac{N}{-M}\right) \pm \sum_{ \pm\left(\frac{N}{-M}\right)}^{\left.\infty\left( \pm \frac{N}{-M}\right)\right)}\left(n f\left( \pm\left(\frac{N}{-M}\right)\right) \ldots . .\right.
$$

Second The Equation For Contraction Nature Of Gravitation

$$
\left.\mathbf{f}\left(\frac{N}{-M}\right) \text { Divide }={ }^{-N} \frac{N}{-M} \cdot+\left(\sum_{\frac{N}{-M}}^{\infty} \frac{N}{-M}\right)\left(+\boldsymbol{n} \boldsymbol{f}\left(\frac{N}{-M}\right)\right)+\sum_{\frac{N}{-M}}^{\infty\left(\frac{N}{-M}\right)}\left(-\boldsymbol{n} \boldsymbol{f}\left(\frac{N}{-M}\right)\right)\right)
$$

10.5 The Combined Field Equation Of Gravitation For Expanding And Contraction Nature

The Combined Field Equation For Gravitation And Its Expanding And Contraction Nature Is The Combination Of Above Mentioned Two Equation

$$
\begin{aligned}
& \mathrm{f}\left(\frac{N}{-M}\right) \text { Attachment }+\mathbf{f}\left(\frac{N}{-M}\right) \text { Divide }=\ldots \ldots . \sum_{\mathrm{f}\left(\frac{N}{-M}\right)}^{\infty\left(\mathrm{f} \frac{N}{-M}\right)}\left(n f\left(\mathrm{~F}\left(\frac{N}{-M}\right)\right)\right) \quad \mp \quad\left(\frac{N}{-M}\right) \pm \sum_{ \pm\left(\frac{N}{-M}\right)}^{\infty\left( \pm\left(\frac{N}{-M}\right)\right)}\left(n f\left( \pm\left(\frac{N}{-M}\right)\right) \ldots . .+\cdots \frac{N}{-M} \cdot+(\right. \\
& \left.\sum_{\frac{N}{-M}}^{\infty\left(\frac{N}{-M}\right)}\left(+\boldsymbol{n} \boldsymbol{f}\left(\frac{N}{-M}\right)\right)+\sum_{\frac{N}{-M}}^{\infty\left(\frac{N}{-M}\right)}\left(-\boldsymbol{n} \boldsymbol{f}\left(\frac{N}{-M}\right)\right)\right) \\
& \text { Now If Sign Varying Terms Cross Each Other Than Remaining Parts Are }
\end{aligned}
$$

$\mathrm{f}\left(\frac{N}{-M}\right)$ Attachment $+\mathbf{f}\left(\frac{N}{-M}\right)$ Divide $=\left(\frac{N}{-M}\right)+\frac{N}{-M}$

For N = -M On Both Sides We Have above Equation In Form

$\mathrm{f}\left(\frac{N}{N}\right)$ Attachment $+\mathbf{f}\left(\frac{N}{N}\right)$ Divide $=\left(\frac{N}{N}\right)+\frac{N}{N}$

$1+1=1+1$

$2=2$

$1=1$

L.H.S = R.H.S

This Proves The Above Equation Of Gravitation Theoretically.

\subsection{Conditions To Find Graviton}

Using The Above Mentioned Conditions We Could Detect Two Types Gravitational Waves And At The Point When Expanding And Contracting Field Combined In Each Other Than Only The Non-Intractable Particle Could Escape From Class Zero Field Or From A Black Hole Because It Has Velocity Higher Than Light And It Has The Matter Emitting Property $(\mathrm{N}=-\mathrm{M})$, This Escaped Non-Intractable Particle Is The Field Quanta Of Gravitational Field "The Graviton". The Identification Of Graviton Is Its Matter Emitting Nature. This Is The Condition To Find Graviton.

\section{Proofs-}

\section{i. Proof Of Space Creation Condition-}

$$
\left(\frac{N}{\left(-\frac{4 \pi V}{3 h^{3}} \cdot \frac{1}{4} \sqrt[8 m^{3} \pi]{\frac{\beta p^{2}}{\beta^{3}}} \cdot e^{\frac{2}{2 m}}\right)}\right)^{2 n}=2 \mathrm{n} \cdot\left(\frac{N}{\left(-\frac{4 \pi V}{3 h^{3}} \cdot \frac{1}{4} \sqrt{\frac{8 m^{3} \pi}{\beta^{3}}} \cdot e^{\frac{\beta p^{2}}{2 m}}\right.}\right)
$$

This is the required condition for the creation of space by the class zero particles and by this these particles attained mass form according to N-I Mechanics.

Now Putting $\mathrm{n}=1 / 2$ and $\mathrm{N}=-\mathrm{M}$ on both sides In Above Equation

$$
\begin{gathered}
\left(\frac{N}{N}\right)^{2 . \frac{1}{2}}=2 \cdot \frac{1}{2}\left(\frac{N}{(N)}\right) \\
1=1 \\
\text { L.H.S }=\text { R.H.S }
\end{gathered}
$$

This proves The Above Equation Of Space Creation.

iii. Proof Of Time Appearance

$$
\text { Now Taking Equation -5(a) }
$$

$\left(\frac{N}{-M}\right)^{2 n-1}=2 \mathrm{n}\left(\frac{N}{-M}\right)^{1-1} \quad$ Taking $\mathbf{n = 1 / 2}$ And N = -M On Both Sides Than We Have

$$
\left(\frac{N}{N}\right)^{2 \frac{1}{2}-1}=2 \frac{1}{2}\left(\frac{N}{N}\right)^{1-1}
$$

$$
\begin{aligned}
(1)^{0} & =(1)^{\mathbf{0}} \\
1 & =1
\end{aligned}
$$

L.H.S = R.H.S

This Proves The Above Condition Of Time Appearance Theoretically

\section{DISCUSSION}

Many theories of physics Satisfy proposed non intractable model universe even if universe expanding w have known half properties of related particle property and very less universe expanding. 
According to above manuscript we can interpret that non intractable particle and disintegrated matter creates infinitely expanded space and almost zero mass Astroparticle, these Astroparticle arrange in the infinitely expanded space and forms River of quantum domains then by the arrangement of these Astroparticle and matter the phenomena of time emerges both the condition of space creation and time appearance are satisfied practically and theoretically.

Then we can say by use of this theory we can expand space and create time as according to the need of situation then we can create many systems to expand these space and time creation conditions and these systems are lab controllable and self controllable by the self moving conscious particles this is far better than artificial intelligence as these systems are depend on energy which is generated by non intractable particle and disintegrated matter and for holding the control on these conditions and situations we could use gravitation as a tool and for generating required gravitation we could generate Graviton in field also by expanding and contracting gravitation and respective gravitational waves we could generate gravitation this work can be done in Ligo and Virgo type labs, The condition to find Graviton is mentioned in above manuscript.

\section{V.CONCLUSION AND FUTURE SCOPE}

The main conclusions of the study may be presented in a short Conclusion Section. In this section, the author(s) should also briefly discuss the limitations of the research and Future Scope for improvement.

Future Scopes are Astrophysical research ,humans nature wuthvuniverse,UNIVERSE IS ALSO SELF CONSCIOUS

Stablishing ultrafast communication speed than the movingvwithoutrsTriction (Non Interactive Mechanics)

\section{REFERENCES}

[1] Nishant Kumar Sharma, Non-Interactive Mechanics, Journal Of Nuclear And Particle Physics, Vol.9 No.1, 2019, pp. 18-41. doi:10.5923/j.jnpp.201909901.03S.

[2] Nishant Kumar Sharma, Law of gravitation for non intractable particles, Research Paper, journal paper(IJSRPAS), vol.7, issue.4 ,pp. 15-22 Aug-2019.

[3] https://www.sciencenews.org/blog/context/new-einstein-equation-wormholes-quantum-gravity 23-04-19

[4] Tayal, D.C, "Nuclear Physics", Himalaya Publishing House, 2017

[5] Kallen.G "Elementary Particle Physics” Addison Weseley,Reading Mass, 1987S.

\section{AUTHORS PROFILE}

Mr. Nishant Kumar Sharma is B.sc,, M.sc . in. Physical Science from Kumaun University, Nainital in 2015, 2017. He is currently working as Independent Researcher and Published Two Articles in international journals which unifies Classical, Relativistic and quantum mechanics, the papers Are Non Interactive mechanics, Law of gravitation for Non Intractable Particles . Article Law of gravitation for Non Intractable Particles is published in ISROSET Journal IJSRPAS. 